\title{
STABILITY OF OPERATOR SEMIGROUPS: IDEAS AND RESULTS
}

\author{
RALPH CHILL \\ Abteilung Angewandte Analysis, Universität Ulm, 89069 Ulm, Germany \\ Current address: Université Paul Verlaine - Metz \\ Laboratoire de Mathématiques et Applications de Metz \\ CNRS, UMR 7122, Bât. A, Ile du Saulcy, 57045 Metz Cedex 1, France \\ E-mail: chill@univ-metz.fr \\ YURI TOMILOV \\ Institute of Mathematics, Polish Academy of Sciences \\ Śniadeckich 8, P.O. Box 21, 00-956 Warszawa, Poland, and \\ Faculty of Mathematics and Computer Science \\ Nicolaus Copernicus University, Chopina 12/18, 87-100 Toruń, Poland \\ E-mail: tomilov@mat.uni.torun.pl
}

1. Introduction. Given a $C_{0}$-semigroup $(T(t))_{t \geq 0}$ on a Banach space $X$ we call an orbit $T(\cdot) x(x \in X)$ stable if

$$
\lim _{t \rightarrow \infty}\|T(t) x\|=0 .
$$

If (1.1) holds for every $x \in X$ then we call $(T(t))_{t \geq 0}$ stable. For single bounded operators, i.e. for discrete operator semigroups, on a Banach space the concept of stability is defined similarly.

It is the purpose of this survey to present a new and unified look on the theory of stability of operator semigroups. Our emphasis is put on the ideas, methods and tools, both for general and concrete semigroups. We give neither a complete overview over existing results nor a historical account on them.

Since the birth of semigroup theory and general operator theory, the theory of stability of operator semigroups has attracted a lot of attention due to several reasons.

First, the theory of stability is important since stable $C_{0}$-semigroups correspond oneto-one to asymptotically stable (in the sense of Lyapunov) well-posed abstract linear

2000 Mathematics Subject Classification: Primary 47D03; Secondary 34D05, 47A10.

The second author was partially supported by a KBN grant.

The paper is in final form and no version of it will be published elsewhere. 
Cauchy problems. The concept of asymptotic stability is fundamental in the theory of ordinary and partial differential equations. This puts stability theory on the ground of (real world) applications.

The theory of stability is also important since stability plays a central role in the structural theory of operators; as examples, we mention the classification of contraction semigroups, invariant subspace theory, similarity and quasisimilarity problems, dilations and functional calculi; see [SNF70], [Bea88], [Kub97], [Atz84], [Kér94], [AE98], [ARS04a], [ARS04b], [AM04].

Second, the theory of stability is rich in what concerns the methods and ideas, and this shall be a main point of this survey. The recent advances deeply interact with modern topics from complex function theory, harmonic analysis, the geometry of Banach spaces, and spectral theory. These interactions lead sometimes to unexpected byproducts: new structure theorems for the invariant subspaces of a Bergman shift [ARS04a], [ARS04b], new maximum principles for harmonic functions [BCT05] or new tauberian theorems [Kor02], [ABHN01], [Chi98], [BNR98b]. This point shows that the theory deserves attention not only from the applied point of view.

Third, the theory of stability is interesting since it has uncovered intimate relations between seemingly unrelated areas and highlighted existing links between different mathematical subjects; see also above.

Finally, the theory of stability is challenging. Despite the definite progress which has been made since the breakthrough papers [AB88] and [LV88], in our opinion the major advances in the theory of stability and the understanding of their place among other mathematical theories (complex function theory, operator theory, partial differential equations) still await their development.

During the last decade, the two surveys [Bat94], [Vũ97] and the two monographs [Nee96], [ABHN01] on the subject have appeared. However, these mainly discuss spectral conditions for stability (see Sections 4 and 5) while we in addition concentrate on conditions involving the boundary behaviour of the resolvent of the generator (Sections 6, 8 and 9). Moreover, we discuss special but important classes of semigroups such as positive semigroups (Section 7), semigroups on Hilbert spaces (Section 8), semigroups on Banach spaces with Fourier type (Section 9), and evolution semigroups (Section 10). We hope that those who already benefited from reading the above mentioned accounts will find quite a few new attractions in this survey. In addition to new aspects in the theory we sometimes give new proofs, interpretations and examples regarding older results. Certainly, all the priorities we set and all the comments reflect our personal point of view. Due to our background most of the results in the survey are formulated for $C_{0}$-semigroups. But with a few exceptions, there are always discrete counterparts. We believe that there should be no difficulty in finding the corresponding statements for discrete semigroups.

Also in order to limit the size of the survey, omissions of several topics were unavoidable. In particular, we do not discuss other types of asymptotic behaviour (e.g. asymptotic almost periodicity) which are from the point of view of applications and techniques of proofs strongly connected to stability. We also do not discuss individual stability and applications of the stability theory to PDEs or to abstract operator theory. 
We have omitted the stability theory of Markov operators (with applications to PDEs), the Katznelson-Tzafriri theory for positive operators (including the $0-2$ law), and nonlinear aspects of stability. We hope to discuss these topics in the future also in connection with the theory presented here.

2. Tools for stability. We need to define or to introduce some concepts or tools which are frequently used in the study of stability of operator semigroups and which will appear throughout this survey.

2.1. Basic notation. Throughout, $X$ will be a (complex) Banach space and $\mathcal{L}(X)$ the space of bounded operators on $X$. A $C_{0}$-semigroup on $X$ is denoted by $\mathcal{T}$ or $(T(t))_{t \geq 0}$, and $A$ is its generator. If $T \in \mathcal{L}(X)$, then $\left(T^{n}\right)_{n \geq 0}$ is the corresponding discrete semigroup. Sometimes, $T$ will be called the generator of this discrete semigroup.

By $X^{*}$ we denote the dual space of $X$, and by $A^{*}, T(t)^{*}$, and $T^{*}$ adjoint operators on the dual space. Given some operator $A$, we let $\varrho(A), \sigma(A), \sigma_{p}(A)$ and $\sigma_{\text {ap }}(A)$ denote the resolvent set, the spectrum, the point spectrum, and the approximative point spectrum, respectively. For every $\lambda \in \varrho(A)$, we let $R(\lambda, A):=(\lambda-A)^{-1}$ be the resolvent of $A$. The kernel and the range of an operator $A$ will be denoted by $\operatorname{Ker} A$ and $\operatorname{Rg} A$, respectively.

2.2. Laplace, Fourier and Carleman transform. For every weakly measurable (weak* measurable, if $X$ is a dual space) function $f: \mathbb{R}_{+} \rightarrow X$ of exponential growth we define the Laplace transform $\hat{f}$ by

$$
\hat{f}(\lambda):=\int_{0}^{\infty} e^{-\lambda t} f(t) d t
$$

for all $\lambda \in \mathbb{C}$ for which the integral above exists in the weak sense (resp. weak* sense). The Laplace transform $\hat{f}$ is analytic in some right half plane of $\mathbb{C}$.

If $f$ is bounded, then $\hat{f}$ is at least analytic in the open right half plane $\mathbb{C}_{+}:=\{\lambda \in$ $\mathbb{C}: \operatorname{Re} \lambda>0\}$. It is well known that if $A$ generates a $C_{0}$-semigroup $(T(t))_{t \geq 0}$, then $\hat{T}(\lambda) x=R(\lambda, A) x$, i.e. the resolvent is the Laplace transform of the semigroup.

For a function $f \in L^{\infty}(\mathbb{R} ; X)$ (or any vector-valued tempered distribution) we denote by $\mathcal{F} f$ the distributional Fourier transform, i.e. $\mathcal{F} f$ is the element of the space $\mathcal{S}^{\prime}(\mathbb{R} ; X):=$ $\mathcal{L}(\mathcal{S}(\mathbb{R}), X)$ of all vector-valued Schwartz distributions which is given by

$$
\langle\mathcal{F} f, \varphi\rangle:=\langle f, \mathcal{F} \varphi\rangle, \quad \varphi \in \mathcal{S}(\mathbb{R}) .
$$

Note that for Schwartz test functions or $L^{1}$ functions $\varphi$, we define the Fourier transform $\mathcal{F} \varphi$ by

$$
\mathcal{F} \varphi(\beta):=\int_{\mathbb{R}} e^{-i \beta t} \varphi(t) d t, \quad \beta \in \mathbb{R}
$$

For every weakly measurable (weak* measurable, if $X$ is a dual space) function $f$ : $\mathbb{R} \rightarrow X$ of subexponential growth (i.e. $\sup _{t \in \mathbb{R}} e^{-\omega|t|}\|f(t)\|<\infty$ for every $\omega>0$ ) we define the Carleman transform $\hat{f}$ by

$$
\hat{f}(\lambda):= \begin{cases}\int_{0}^{\infty} e^{-\lambda t} f(t) d t, & \operatorname{Re} \lambda>0 \\ -\int_{-\infty}^{0} e^{-\lambda t} f(t) d t, & \operatorname{Re} \lambda<0\end{cases}
$$


The Carleman transform $\hat{f}$ is analytic in $\mathbb{C} \backslash i \mathbb{R}$. The set of singular points of $\hat{f}$ on $i \mathbb{R}$ will be called the Carleman spectrum of $f$. There will be no ambiguity in the notation of the Laplace and the Carleman transform. For the theory of Laplace and Carleman transforms we refer to [ABHN01] and [Prü93], for that of Fourier transforms to [Sch57, Sch58].

2.3. Fractional powers of sectorial operators. We call a closed, densely defined operator $A$ sectorial if there exists $\theta \in(0, \pi)$ such that the spectrum of $A$ is contained in the closure of the sector $\Sigma_{\theta}:=\{\lambda \in \mathbb{C}:|\arg \lambda|<\theta\}$ and such that $\lambda R(\lambda, A)$ is uniformly bounded outside every larger sector $\Sigma_{\theta^{\prime}}$. For every sectorial operator $A$ and every holomorphic function $f: \Sigma_{\theta^{\prime}} \rightarrow \mathbb{C}$ satisfying $|f(\lambda)| \leq M\left|\frac{1+\lambda^{2}}{\lambda}\right|^{s}(s>0)$ one can define a closed operator $f(A)$ by the formula

$$
f(A):=\left(f \varphi^{k}\right)(A)\left(A(I+A)^{-2}\right)^{-k},
$$

where $\varphi(\lambda)=\frac{\lambda}{(1+\lambda)^{2}}$ and $k \in \mathbb{N}$ is chosen large enough so that the integral

$$
\left(f \varphi^{k}\right)(A):=\frac{1}{2 \pi i} \int_{\partial \Sigma_{\theta^{\prime}}} f(\lambda) \varphi^{k}(\lambda) R(\lambda, A) x d \lambda
$$

converges absolutely. Using this functional calculus, one obtains for $f(\lambda)=\lambda^{\gamma}$ the fractional power $A^{\gamma}(\gamma \in \mathbb{R})$, and it is clear that $A^{\gamma} A^{\nu}=A^{\gamma+\nu}$ when suitably interpreted; see [McI86], [Haa06]. This definition of fractional powers is consistent with the classical definitions from [Bal60], [Kom66], or [MS01, Definition 3.1.1].

If $A$ generates a bounded $C_{0}$-semigroup, then for every $\beta \in \mathbb{R}$ the operator $i \beta-A$ is sectorial, and therefore the fractional powers $(i \beta-A)^{\gamma}$ are well defined. Note that $\operatorname{Rg}(i \beta-A)^{\gamma} \supset \operatorname{Rg}(i \beta-A)^{\nu}$ whenever $\nu>\gamma$. This inclusion is strict in general.

If $\lambda \in \mathbb{C}_{+}$and $\gamma>0$, then, by [MS01, Lemma 6.1.5], we also have the representation

$$
R(\lambda, A)^{\gamma} x=\frac{1}{\Gamma(\gamma)} \int_{0}^{\infty} e^{-\lambda t} t^{\gamma-1} T(t) x d t, \quad \operatorname{Re} \lambda>0, x \in X,
$$

By this representation, $(\operatorname{Re} \lambda)^{\gamma} R(\lambda, A)^{\gamma}$ is a fractional Abel mean of the semigroup $\left(e^{-i \operatorname{Im} \lambda t} T(t)\right)_{t \geq 0}$ for fixed $\operatorname{Im} \lambda$ (see also the mean ergodic theorem below).

Fractional powers will play a crucial role in relating stability conditions for semigroups on Banach spaces to the Fourier type of these spaces; see Sections 8 and 9 below.

2.4. Ergodic theory. Given $f \in L^{\infty}\left(\mathbb{R}_{+} ; X\right)$, we call $\left(\lambda^{\gamma} / \Gamma(\gamma)\right) \int_{0}^{\infty} e^{-\lambda t} t^{\gamma-1} f(t) d t, \lambda>0$, the (fractional) Abel mean of order $\gamma>0$. It is well known (see [ABHN01, Chapter 4]) that if $\lim _{t \rightarrow \infty} f(t)=f_{\infty}$ exists, then the Abel means of any order converge to the same limit $f_{\infty}$ as $\lambda$ tends to $0+$.

Consequently, if a $C_{0}$-semigroup is stable, then the Abel means converge to zero. Since the Laplace transform of the semigroup is the resolvent of the generator, the convergence of Abel means can be expressed in terms of properties of the resolvent or of spectral properties of the generator. This gives simple necessary stability conditions and shows close ties between stability and mean ergodicity of semigroups.

These necessary stability conditions are expressed in the following mean ergodic theorem which deals also with fractional Abel means. The general case $(\gamma \neq 1)$ has been 
proved in [Kom69, Proposition 2.3, Theorem 2.4], [Wes98, Theorem 2.4]. For the classical case $\gamma=1$, see for example [Yos78, Section VIII.4] or [ABHN01, Corollary 4.3.2].

Theorem 2.1 (Mean Ergodic Theorem). Let $A$ be the generator of a bounded $C_{0}$-semigroup on a Banach space $X$, and let $\gamma>0$. Then:

(1) The following assertions are equivalent.

(i) $\lim _{\lambda \rightarrow 0} \lambda^{\gamma} R(\lambda, A)^{\gamma}=: P$ exists strongly.

(ii) $X=\operatorname{Ker} A \oplus \overline{\operatorname{Rg} A}$.

If (i) and (ii) hold, then $P$ is the projection onto $\operatorname{Ker} A$ along $\overline{\operatorname{Rg} A}$.

If $X$ is reflexive, then (i) and (ii) are always true.

(2) $\lim _{\lambda \rightarrow 0} R(\lambda, A)^{\gamma} x=: y$ exists if and only if $x \in \operatorname{Rg}(-A)^{\gamma}$, and in this case $y \in$ $D\left((-A)^{\gamma}\right) \cap \overline{\operatorname{Rg}(A)}$ and $x=(-A)^{\gamma} y$.

A semigroup $(T(t))_{t \geq 0}$ satisfying either of the equivalent conditions (1)(i) or (1)(ii) will be called mean ergodic. We note that the relations between fractional powers of (bounded) operators and ergodic properties of their corresponding discrete semigroups were recently thoroughly investigated in [DL01].

Since a semigroup $(T(t))_{t \geq 0}$ is stable if and only if $\left(e^{i \beta t} T(t)\right)_{t \geq 0}$ is stable for all $\beta \in \mathbb{R}$, the mean ergodic theorem (or a simple direct reasoning) implies the following.

Corollary 2.2. Let $A$ be the generator of a stable $C_{0}$-semigroup on $X$. Then $\operatorname{Rg}(i \beta-A)$ is dense in $X$ for every $\beta \in \mathbb{R}$ or, equivalently, $\sigma_{p}\left(A^{*}\right) \cap i \mathbb{R}$ is empty.

Note that for bounded $C_{0}$-semigroups, $\sigma_{p}(A) \cap i \mathbb{R} \subset \sigma_{p}\left(A^{*}\right) \cap i \mathbb{R}[\mathrm{AB} 88$, Lemma 2.3], and the two sets are equal if $X$ is reflexive.

2.5. Edge-of-the wedge theorems. It has been shown recently in several articles how criteria for the analytic extendability of analytic functions across a linear boundary can be applied in order to obtain stability. Such criteria are provided by so-called edge-of-thewedge theorems. In the classical edge-of-the-wedge theorem the equality of distributional boundary values of analytic functions defined on the two sides of a linear boundary suffices for obtaining analytic extendability. However, for the study of stability, more subtle edge-of-the-wedge theorems dealing only with pointwise boundary values are needed. First theorems of this kind have been proved in [Wol47, Theorem D] and [Car44]; see also [Tom01, Theorem 4.4] for a corrected version of [Wol47, Theorem D] with a different proof. These results were extended in [CT04, Theorem 3.1] and finally improved to the following version from [BCT05, Theorem 5.4]. It is of independent interest for complex function theory.

In order to formulate the theorem, we define the rectangle

$$
R:=\{z \in \mathbb{C}:-1 \leq \operatorname{Re} z \leq 1,-1 \leq \operatorname{Im} z \leq 1\},
$$

and for $\theta \in\left(0, \frac{\pi}{2}\right)$ we let

$$
\Sigma_{\theta}:=\{z \in \mathbb{C}: \theta<\arg z<\pi-\theta\} .
$$

THEOREM 2.3 (Edge-of-the-wedge). Let $f: R \backslash \mathbb{R} \rightarrow \mathbb{C}$ be analytic, and define $F: R \backslash \mathbb{R} \rightarrow$ $\mathbb{C}$ by $F(z)=f(z)-f(\bar{z})(z \in R \backslash \mathbb{R})$. Assume that 
(1) there exists a constant $m \geq 0$ such that

$$
\sup _{\alpha \in(-1,1)}|f(\alpha+i \beta)|=O\left(|\beta|^{-m}\right), \quad \beta \rightarrow 0,
$$

(2) there exist a measurable function $G: R \backslash \mathbb{R} \rightarrow \mathbb{R}_{+}$and a continuous function $H: R \backslash \mathbb{R} \rightarrow \mathbb{R}_{+}$such that $|F| \leq G \cdot H$,

$$
\sup _{\beta \in(0,1)}\|G(\cdot+i \beta)\|_{L^{1}}<\infty,
$$

and there exists $\theta_{0} \in\left(0, \frac{\pi}{2}\right)$ such that

$$
\lim _{\substack{z \rightarrow \alpha \\ z \in \alpha+\Sigma_{\theta_{0}}}} H(z)=0 \quad \text { for every } \alpha \in(-1,1) .
$$

Then the function $f$ admits an analytic extension to $R$.

2.6. Two examples. Throughout this survey most of the statements will be illustrated on the following two basic examples of stable semigroups. The examples were used for the study of stability in [BNR98a], [dLVW02] and [CT03]. Choosing appropriate weights allows one to check for optimality of the theorems presented below.

EXAMPLE 2.4. In the first example, choose $\omega: \mathbb{R}_{+} \rightarrow(0, \infty)$ a continuous and nonincreasing function such that

(i) $\lim _{t \rightarrow+\infty} \omega(t)=0$, and

(ii) the function $1 / \omega$ is of subexponential growth on $\mathbb{R}_{+}$.

Let $X_{p}=L^{p}\left(\mathbb{R}_{+} ; \omega(t) d t\right)(1 \leq p<\infty)$, and consider the right-shift $C_{0}$-semigroup $(S(t))_{t \geq 0}$ defined by

$$
(S(t) f)(s):=\left\{\begin{array}{ll}
f(s-t), & s \geq t \geq 0, \\
0, & 0 \leq s<t,
\end{array} \quad f \in X_{p} .\right.
$$

An easy calculation shows that the semigroup $(S(t))_{t \geq 0}$ is stable.

EXAMPLE 2.5. In the second example, choose the weight $\omega: \mathbb{R}_{+} \rightarrow(0, \infty)$ as in Example 2.4 and extend it by $\omega(0)$ on $\mathbb{R}_{-}$. Let $X_{p}:=L^{p}(\mathbb{R} ; \omega(t) d t)(1 \leq p<\infty)$. We define the right-shift $C_{0}$-group $(S(t))_{t \in \mathbb{R}}$ on $X_{p}$ :

$$
(S(t) f)(s):=f(s-t), \quad s, t \in \mathbb{R}, f \in X_{p} .
$$

As in Example 2.4, the operators $S(t)$ are contractive for every $t \in \mathbb{R}_{+}$, and the semigroup $(S(t))_{t \geq 0}$ is stable. Note that the group $(S(t))_{t \in \mathbb{R}}$ is of subexponential growth. If $1 / \omega$ is polynomially bounded, then $(S(t))_{t \in \mathbb{R}}$ will also be polynomially bounded, and the generator $D$ is then a generalised scalar in view of the estimate $\|R(\lambda, D)\| \leq C /|\operatorname{Re} \lambda|^{m}$ for some $m \in \mathbb{N}$ and $\lambda \in \mathbb{C} \backslash i \mathbb{R}$.

3. Orbits and stability. In general, we are interested in conditions on the generator $A$ which imply stability of the corresponding semigroup. This interest is motivated by the applications to Cauchy problems in which the generator $A$ is given but in general not the semigroup. Nevertheless, there are some other important and equally interesting 
stability conditions in terms of the semigroup itself. This section is devoted to present these (nonspectral and nonalgebraic) stability conditions.

3.1. Limit isometric group. A bounded semigroup $(T(t))_{t \geq 0}$ is stable if and only if the algebraic factor space $X / X_{0}$ is trivial, where $X_{0}:=\{x \in X: T(\cdot) x$ is stable $\}$. This very obvious remark provides a way of proving stability which has indeed often been used in the literature. The idea behind it, i.e. factoring out a good part of a given set and showing that the factor is zero, is in fact a frequent idea in mathematics. In the special case here, this idea leads to the so-called limit isometric group, an efficient device for proving stability of operator semigroups.

The origin of this operator-theoretical construction can be traced back to Dixmier [Dix50], Sz.-Nagy [SN47] and Sz.-Nagy \& Foiaş [SNF70]; see also [Gha75]. It has been modified by Sklyar \& Shirman [SS82], and it was extended and refined by many authors since then. The following formulation is due to Bercovici [Ber93, Theorem 2 and p. 64] although it was surely known before to other researchers in the domain.

Theorem 3.1. Let $(T(t))_{t \geq 0}$ be a bounded $C_{0}$-semigroup on a Banach (resp. Hilbert) space $X$. Then there exist a Banach (resp. Hilbert) space $Y$, an isometric (resp. unitary) $C_{0}$-group $(S(t))_{t \in \mathbb{R}}$ on $Y$ and a bounded linear operator $\pi: X \rightarrow Y$ such that

(i) $S(t) \pi=\pi T(t), t \geq 0$;

(ii) $\pi(x)=0$ if and only if $\lim _{t \rightarrow \infty}\|T(t) x\|=0$;

(iii) $Y=\overline{\bigcup_{t \geq 0} S(-t) \pi(X)}$.

The group $(S(t))_{t \in \mathbb{R}}$ is unique up to similarity.

In the literature devoted to stability, Theorem 3.1 was used mostly in the Banach space context and in a version when $(S(t))_{t \geq 0}$ is just an isometric semigroup. The important fact that $(S(t))_{t \geq 0}$ can always be chosen as a group (with some additional spectral properties) was observed in [BG94, Proposition 2.1]. It follows from Douglas' extension theorem [Dou69, Theorem 1].

If $A$ is the generator of $(T(t))_{t \geq 0}$ and $B$ the generator of $(S(t))_{t \in \mathbb{R}}$ then one can show that $\sigma(B) \subset \sigma(A) \cap i \mathbb{R}$, so that information about the boundary spectrum of $A$ yields information about the spectrum of $B$. This spectral inclusion allows one to apply the well-developed local spectral theory for isometries in order to prove that $Y$ is trivial, i.e. that the semigroup $(T(t))_{t>0}$ is stable. The latest developments of this idea can be found in [BNR98a], [BNR98b], [Bat96].

The above spectral approach can be put in a more general framework. A method of local resolvent estimates of $B$ in terms of those of $A$ and their application to stability was recently developed in [Tom01]. It is most efficient when $X$ is a Hilbert space.

3.2. Complete trajectories. Besides the approach via limit isometric groups or semigroups, there is another but very similar approach to proving stability of a given semigroup. This approach even allows us to study stability of operator families more general than semigroups, e.g. evolution families.

We call a function $F: \mathbb{R} \rightarrow X$ a complete trajectory for a $C_{0}$-semigroup $(T(t))_{t \geq 0}$ if for all $t \geq 0$ and all $s \in \mathbb{R}: F(t+s)=T(t) F(s)$. The following theorem characterises 
stability in terms of nonexistence of bounded, nontrivial complete trajectories for the adjoint semigroup. A proof can be found in [Lin71, Theorem 4.3] or [Der76, Théorème 2] for the discrete case, and in [BBG96, Theorem 3.1] or [Vũ93] for the continuous case. A similar result is even true for bounded evolution families (see [BCT02] or Theorem 10.1 below).

THEOREM 3.2. For a bounded $C_{0}$-semigroup $(T(t))_{t \geq 0}$ on a Banach space $X$ the following statements are equivalent:

(i) The semigroup $(T(t))_{t \geq 0}$ is stable,

(ii) The adjoint semigroup $\left(T(t)^{*}\right)_{t \geq 0}$ does not admit a bounded, nontrivial complete trajectory.

(iii) If $B^{*}$ denotes the unit ball in $X^{*}$, then $\bigcap_{t \geq 0} T(t)^{*} B^{*}=\{0\}$.

The following statement is analogous to Theorem 3.2 and characterises convergent semigroups in terms of mean ergodicity and complete trajectories.

TheOREM 3.3. For a bounded $C_{0}$-semigroup $(T(t))_{t \geq 0}$ on a Banach space $X$ the following statements are equivalent:

(i) $(T(t))_{t \geq 0}$ is convergent,

(ii) $(T(t))_{t \geq 0}$ is mean ergodic, and the only bounded, nontrivial complete trajectories for the adjoint semigroup $\left(T(t)^{*}\right)_{t \geq 0}$ are constants.

(iii) $(T(t))_{t \geq 0}$ is mean ergodic, and $\bigcap_{t \geq 0} T(t)^{*} B^{*}=\operatorname{Ker} A^{*}$.

The proof of Theorem 3.3 is a simple adaptation of the proof of Theorem 3.2 from [Lin71, Theorem 4.3]. Clearly, if $X$ is reflexive then the assumption of mean ergodicity can be omitted from (ii) and (iii). A statement related to Theorem 3.3 was proved in [Rub77, Theorem 10].

REMARK 3.4. Note that stable semigroups may have nontrivial bounded complete trajectories, and that non-stable semigroups may have no such trajectories. One may take for examples the (stable) left-shift semigroup on $L^{2}\left(\mathbb{R}_{+}\right)$and its (nonstable) adjoint right-shift semigroup. Thus the use of the adjoint semigroup in Theorem 3.2 is essential.

We collect some basic properties of bounded complete trajectories for $\left(T(t)^{*}\right)_{t \geq 0}$ in the following proposition.

Proposition 3.5. Let $(T(t))_{t \geq 0}$ be a bounded $C_{0}$-semigroup on a Banach space $X$ with generator $A$, let $F$ be a bounded complete trajectory for $\left(T(t)^{*}\right)_{t \geq 0}$, and let $\hat{F}$ be its Carleman transform. Then:

(i) For every $\lambda \in \mathbb{C}_{+}$and every $\mu \in \mathbb{C} \backslash i \mathbb{R}$, the following identity holds:

$$
\hat{F}(\mu)=R\left(\lambda, A^{*}\right) F(0)+(\lambda-\mu) R\left(\lambda, A^{*}\right) \hat{F}(\mu) .
$$

(ii) If $\sigma_{C}(F)$ is the Carleman spectrum of $F$, then

$$
\sigma_{C}(F) \subset-i \sigma(A) \cap \mathbb{R} .
$$


The proof of the property (i) can be found in [BCT02, Lemma 6.1], while the property (ii) follows directly from an argument given in [BCT02, p. 133]; see also [Vũ93, Proposition 3.7] where (ii) was proved in a particular case.

The Carleman transform of a bounded complete trajectory for the adjoint semigroup and the Carleman spectrum play the roles of the resolvent and the spectrum of the generator of the limit isometric group associated with a bounded semigroup. The fact that also the Carleman spectrum of the complete trajectory cannot be larger than the boundary spectrum of the generator (compare with the corresponding property of the limit isometric group) becomes especially useful when dealing with spectral stability criteria. Observe that the inclusion (3.2) is strict, in general.

Since the Carleman spectrum of a bounded nonzero function is nonempty, the equivalence $(\mathrm{i}) \Leftrightarrow$ (ii) of Theorem 3.2 allows one to prove stability by function-theoretic methods. That is, a semigroup is stable if and only if for every bounded complete trajectory $F$ of the adjoint semigroup the Carleman transform $\hat{F}$ extends to an entire function (which is eventually zero). This observation links the study of stability with the theory of analytic continuation of functions across a linear boundary, and lies at the heart of many results presented here.

3.3. Decay rates of orbits. In general, the structure of the orbits of a stable semigroup can be very complicated. For example, the orbits may decay arbitrarily slowly, [Mül88].

THEOREM 3.6 (Müller). Let $\left(T^{n}\right)_{n \geq 0}$ be a stable semigroup on a Banach space $X$ such that $\sigma(T) \cap \mathbb{T} \neq \emptyset$. Then, for every positive sequence $\left(a_{n}\right)_{n \in \mathbb{N}} \in c_{0}$ there exists an $x \in X$ such that $\left\|T^{n} x\right\| \geq a_{n}$ for every $n \geq 0$.

For variants of this statement for weak orbits and with large sets of slowly decaying orbits see [Mül01], [Mül03]. For a continuous version of Theorem 3.6, i.e. a version for stable but not exponentially stable $C_{0}$-semigroups, see [Nee96, Lemma 3.1.7].

3.4. Supercyclicity. We call a vector $x \in X$ supercyclic for an operator $T \in \mathcal{L}(X)$ if the set $\left\{c T^{n} x: n \geq 0, c \in \mathbb{C}\right\}$ is dense in $X$. An operator $T$ is, by definition, supercyclic if it has at least one supercyclic vector.

Note that $T$ is supercyclic if and only if $\lambda T$ is supercyclic for every $\lambda \in \mathbb{C} \backslash\{0\}$. The following surprising theorem has been proved in [AB97, Theorem 2.3].

THEOREM 3.7 (Ansari-Bourdon). If $T$ is power bounded and supercyclic, then $\left(T^{n}\right)_{n \geq 0}$ is stable.

Supercyclicity of bounded semigroups seems to be a much stronger property than stability. It was shown recently in [GM04] and [LSPL03] that the Volterra operator $(V f)(t)=\int_{0}^{t} f(t) d t$ is not supercyclic on $L^{2}(0,1)$. At the same time, $\sigma(V)=\{0\}$, and so the powers of $V$ tend to zero exponentially. In general, supercyclicity imposes strong restrictions on the spectral and geometric properties of an operator. In particular, normal operators cannot be supercyclic, and also the point spectrum of the adjoint of a supercyclic operator may contain at most one point.

A variant of Theorem 3.7 in the context of $C_{0}$-semigroups was recently obtained in [Kér05]: if $(T(t))_{t \geq 0}$ is bounded and admits a supercyclic vector (i.e. a vector $x \in X$ 
such that $\{c T(t) x: t \geq 0, c \in \mathbb{C}\}$ is dense in $X)$, then $(T(t))_{t \geq 0}$ is stable under the additional assumption that $\overline{\operatorname{Rg} T(t)}=X, t \geq 0$. The question whether this assumption can be removed remains open.

3.5. Real integrability conditions. The Datko-Pazy theorem characterising exponential stability of semigroups ([Dat72]) admits the following version for stability of individual orbits. Note that there is no growth restriction on the semigroup. The result is an immediate consequence of the inequality $\|T(t+s) x\| \leq C\|T(t) x\|, s \in[0,1]$, following from the semigroup property.

Theorem 3.8 (Datko, Pazy). Let $(T(t))_{t \geq 0}$ be a $C_{0}$-semigroup, and let $x \in X$. If

$$
\int_{0}^{\infty}\|T(t) x\|^{p} d t<\infty \quad \text { for some } p \in[1, \infty)
$$

then the orbit $T(\cdot) x$ is stable.

By considering the semigroup from Example 2.4 with an appropriate weight $w$ one can see that there are stable semigroups for which (3.3) does not hold for any $x \in X$. For more general conditions of type (3.3) see [Nee02].

The following integrability condition, however, characterises stable orbits of bounded semigroups.

Proposition 3.9. If $(T(t))_{t \geq 0}$ is a bounded $C_{0}$-semigroup on a Banach space $X$ and $p \in[1, \infty)$, then $T(\cdot) x$ is stable if and only if

$$
\lim _{t \rightarrow \infty} \int_{t}^{a t} \frac{\|T(s) x\|^{p}}{s} d s=0 \quad \text { for every } a>0 .
$$

While the forward implication follows from [Dra70, Theorem B], the backward implication is a simple consequence of the semigroup property as in the above Datko-Pazy result. Proposition 3.9 was stated in [CT03, p. 509] with the difference that only the existence of the limit in (3.4) for every $a>0$ was required; but then the statement becomes false unless the limit is independent of $a>0$.

Finally we give a real integrability condition pertaining to improper integrability of semigroup orbits.

Proposition 3.10. Let $(T(t))_{t \geq 0}$ be a bounded $C_{0}$-semigroup on a Banach space $X$, and let $x \in X$. If

$$
\int_{0}^{\infty} T(t) x d t \quad \text { exists }
$$

then $T(\cdot) x$ is stable.

Proof. Observe that for every $s>0$ and every $x \in X$

$$
\int_{0}^{\infty} T(t) x d t=\sum_{n=0}^{\infty} \int_{n s}^{(n+1) s} T(t) x d t=\sum_{n=0}^{\infty} T(s)^{n} \int_{0}^{s} T(t) x d t .
$$

Hence, $\int_{0}^{s} T(t) x d t \in X_{0}:=\{y \in X: T(\cdot) y$ is stable $\}$ for every $s>0$, and since $X_{0}$ is a closed subspace, we obtain that $x=\lim _{s \rightarrow 0+} \frac{1}{s} \int_{0}^{s} T(t) x d t \in X_{0}$. 
The converse implications in Theorem 3.8 and Proposition 3.10 clearly do not hold; consider the left shift semigroup on $C_{0}\left(\mathbb{R}_{+}\right)$for a counterexample.

4. Countable spectrum conditions and stability. In this section we turn to spectral conditions for stability. They are the easiest to check and thus perhaps the most interesting for applications to Cauchy problems.

By the mean ergodic theorem, it is the boundary spectrum of the generator, i.e. the spectrum on the imaginary axis (resp. on the unit circle in the discrete case) which plays a central role when studying stability. The main question in this section is whether the spectrum alone (nature of the spectrum, size of the spectrum) already determines stability.

We will see that if $A$ generates a bounded $C_{0}$-semigroup and if

- the boundary spectrum $\sigma(A) \cap i \mathbb{R}$ is empty, or

- the boundary spectrum is countable and contains no residual spectrum (ArendtBatty-Lyubich-Vu Theorem),

then the semigroup is stable. In many applications, the countable spectrum condition is satisfied with only one point in the boundary spectrum, e.g. when $A$ generates a bounded holomorphic or positive eventually norm-continuous semigroup.

4.1. Empty boundary spectrum. We present the special case of empty boundary spectrum separately. It is an immediate consequence of Ingham's tauberian theorem for which we present a short and instructive proof. Later we will indicate how this proof can be adapted in order to prove also the ABLV theorem or the Katznelson-Tzafriri theorem (see Corollary 4.4 and Theorem 5.2 below), thus unifying the proofs of these major results in the theory of stability.

THEOREM 4.1 (Ingham). Let $f \in B U C\left(\mathbb{R}_{+} ; X\right)$ (the space of bounded, uniformly continuous, $X$-valued functions on $\mathbb{R}_{+}$) be such that its Laplace transform $\hat{f}$ has a locally integrable extension on the imaginary axis in the sense that $(\hat{f}(\alpha+i \cdot))_{\alpha \searrow 0}$ converges in $L_{\text {loc }}^{1}(\mathbb{R} ; X)$ to some function $\hat{f}(i \cdot)$. Then $f \in C_{0}\left(\mathbb{R}_{+} ; X\right)$.

Proof. Let $\varphi \in \mathcal{S}(\mathbb{R})$ be such that $\mathcal{F} \varphi \in \mathcal{D}(\mathbb{R})$. Then, by Plancherel's theorem,

$$
\begin{aligned}
f * \varphi(t) & =\int_{0}^{\infty} f(s) \varphi(t-s) d s=\lim _{\alpha \rightarrow 0+} \int_{0}^{\infty} e^{-\alpha s} f(s) \varphi(t-s) d s \\
& =\lim _{\alpha \rightarrow 0+} \int_{\mathbb{R}} \hat{f}(\alpha+i \beta) e^{i \beta t} \mathcal{F}^{-1} \varphi(\beta) d \beta=\int_{\mathbb{R}} \hat{f}(i \beta) e^{i \beta t} \mathcal{F}^{-1} \varphi(\beta) d \beta .
\end{aligned}
$$

By the Lemma of Riemann-Lebesgue,

$$
\lim _{|t| \rightarrow \infty} f * \varphi(t)=0 .
$$

Choosing an approximate unit of appropriate test functions and using that $f$ is bounded and uniformly continuous, one obtains the claim.

Applying Ingham's theorem to each orbit $T(\cdot) x$, the following corollary is immediate. However, note that this corollary also easily follows from Proposition 3.5 and Theorem 3.2. 
Corollary 4.2. If $A$ generates a bounded $C_{0}$-semigroup and if the boundary spectrum $\sigma(A) \cap i \mathbb{R}$ is empty, then the semigroup is stable.

4.2. Countable boundary spectrum. The case of countable boundary spectrum (the ABLV theorem) can also be proved using a tauberian theorem. The corresponding tauberian theorem is a generalisation of Ingham's theorem. One allows for some singularities of the Laplace transform $\hat{f}$ on the imaginary axis, but imposes an additional ergodic condition on $f$, and obtains the same conclusion. The following formulation of the theorem can be first found in [BNR98b], but the proof we give is taken from [Chi98]. It uses Loomis' theorem on almost periodic functions; a relatively short proof of Loomis' theorem can be found in Katznelson [Kat68, Theorem 5.21]. In more abstract forms, a proof of the following theorem can also be found in [Bas95], [Bas79], [CP01], [CF02].

THEOREM 4.3. Let $f \in B U C\left(\mathbb{R}_{+} ; X\right)$ be such that its Laplace transform $\hat{f}$ has a continuous extension to $i(\mathbb{R} \backslash E)$, where $E \subset \mathbb{R}$ is a closed and countable set. If, for every $\beta \in E$,

$$
\lim _{\alpha \rightarrow 0+} \alpha \hat{f}_{s}(\alpha+i \beta)=0 \text { uniformly in } s \in \mathbb{R}_{+},
$$

where $f_{s}(t)=f(s+t)$, then $f \in C_{0}\left(\mathbb{R}_{+} ; X\right)$.

Proof. We define the operator

$$
K: L^{1}(\mathbb{R}) \rightarrow B U C(\mathbb{R} ; X) / C_{0}(\mathbb{R} ; X)=: Z, \quad \varphi \mapsto f * \varphi+C_{0}(\mathbb{R} ; X),
$$

in which we have extended the function $f$ by 0 for negative reals. Similarly as in the proof of Ingham's theorem, one shows that for every $\varphi \in \mathcal{S}(\mathbb{R})$ such that $\mathcal{F} \varphi \in \mathcal{D}(\mathbb{R})$ and $\operatorname{supp} \mathcal{F}^{-1} \varphi \cap E=\emptyset, f * \varphi \in C_{0}(\mathbb{R} ; X)$, i.e. $K \varphi=0$. Hence, for every $z^{*} \in Z^{*}$ the function $K^{*} z^{*} \in L^{\infty}(\mathbb{R})$ has spectrum contained in $-E$, i.e. its Fourier transform has support contained in $-E$.

Since $E$ is countable, for every $\psi \in L^{1}(\mathbb{R})$ the function $K^{*} z^{*} * \psi$ is almost periodic by Loomis' theorem [Kat68]. From the ergodic condition (4.1) one easily deduces that the Abel means $\alpha \widehat{K^{*} z^{*}}(\alpha-i \beta)$ converge to zero as $\alpha \rightarrow 0+$ for all $\beta \in E$. By the uniqueness theorem for almost periodic functions [LZ82, Section 2.3], $K^{*} z^{*}=0$ for every $z^{*} \in Z^{*}$. Hence, $K=0$, i.e. $f * \varphi \in C_{0}(\mathbb{R} ; X)$ for every $\varphi \in L^{1}(\mathbb{R})$. Choosing an approximate unit of $L^{1}$ functions and using that $f$ is bounded and uniformly continuous, one obtains the claim.

Applying Theorem 4.3 to each orbit $T(\cdot) x$ and noting that the spectral condition $\overline{\operatorname{Rg}(i \beta-A)}=X$ implies the ergodicity condition (4.1), we obtain as a corollary the ABLV theorem. This theorem has been proved independently in [AB88] and [LV88]. While in [LV88] the ABLV theorem was proved by a pure semigroup method, the article [AB88] already emphasises the tauberian character. However, the proof of the corresponding tauberian statement was quite complicated using an argument of transfinite induction. It is remarkable that the ABLV theoem can be also deduced from results in [Bas79] and [Atz84]. The ABLV theorem is also a corollary to the Katznelson-Tzafriri theorem below and to a recent stability result involving resolvent conditions (see Theorem 6.2 below). Besides the present proof based on Theorem 4.3 we will thus indicate two more proofs of the ABLV theorem. 
Corollary 4.4 (ABLV). If A generates a bounded $C_{0}$-semigroup on a Banach space $X$, if the boundary spectrum $\sigma(A) \cap i \mathbb{R}$ is countable, and if $\operatorname{Rg}(i \beta-A)$ is dense in $X$ for every $\beta \in \mathbb{R}$, then the semigroup is stable.

The countable spectrum condition is the best possible condition among the spectral conditions if no other than spectral assumptions are made. A standard counterexample is the unitary group $U(t) f(s)=e^{t s} f(s)$ on $L^{2}(E ; d \mu)$, where $E \subset i \mathbb{R}$ is closed and uncountable and $\mu$ is a nonatomic measure on $E$.

Note on the other hand that for any given closed set $E \subset \mathbb{R}$ there exists a stable semigroup whose generator has boundary spectrum $i E$. One may, for instance, consider multiplication semigroups on appropriate $L^{2}$ spaces in order to see this. In Examples 2.4 and 2.5, the boundary spectrum is the whole imaginary axis. This shows that spectral conditions as considered above are on the one hand best possible, but on the other hand far from characterising stable semigroups.

\section{The Katznelson-Tzafriri theorem and stability}

5.1. The Katznelson-Tzafriri theorem. Motivated by studies of differences of powers of positive operators, Katznelson \& Tzafriri discovered in [KT86] a new kind of tauberian theorem for power series. We only formulate the corresponding result in the operator context.

Denoting by $A(\mathbb{T})$ and $A^{+}(\mathbb{T})$ the spaces of Fourier transforms of sequences in $l^{1}(\mathbb{Z})$ and $l^{1}(\mathbb{N})$, respectively, we say that a function $f \in A^{+}(\mathbb{T})$ is of spectral synthesis with respect to a closed set $E \subset \mathbb{T}$, if there exists a sequence $\left(f_{n}\right)_{n \in \mathbb{N}} \subset A(\mathbb{T})$ such that each $f_{n}$ vanishes in a neighbourhood of $E$ and $\lim _{n \rightarrow \infty} f_{n}=f$ in $A(\mathbb{T})$.

Given $f=\mathcal{F} a \in A^{+}(\mathbb{T})$ with $a \in l^{1}(\mathbb{N})$ and given a power bounded $T \in \mathcal{L}(X)$, we define $f(T):=\sum_{n=0}^{\infty} a_{n} T^{n}$.

Theorem 5.1 (Katznelson-Tzafriri). Let $T \in \mathcal{L}(X)$ be a power bounded operator. If $f \in A^{+}(\mathbb{T})$ is of spectral synthesis with respect to the boundary spectrum $\sigma(T) \cap \mathbb{T}$ then $\lim _{n \rightarrow \infty}\left\|T^{n} f(T)\right\|=0$.

One may interpret the Katznelson-Tzafriri theorem as an individual stability result, i.e. $\lim _{n \rightarrow \infty} T^{n} x=0$ for every $x \in \operatorname{Rg} f(T)$ and every appropriate $f$, and at the same time it gives a uniform estimate for the convergence to 0 . If $\operatorname{Rg} f(T)$ is dense in $X$, then the Katznelson-Tzafriri theorem even implies stability of $\left(T^{n}\right)_{n \geq 0}$.

The remark by J. Bourgain in [KT86] shows that Theorem 5.1 is a characterisation of $f$ being of spectral synthesis in the following sense: $f \in A^{+}(\mathbb{T})$ is of spectral synthesis with respect to a closed set $E \subset \mathbb{T}$ if and only if for every contraction $T$ on an arbitrary Banach space with $\sigma(T) \subset E$ we have $\lim _{n \rightarrow \infty}\left\|T^{n} f(T)\right\|=0$.

Under the assumptions of the Katznelson-Tzafriri theorem, $\sigma(T) \cap \mathbb{T} \subset\{\lambda \in \mathbb{T}$ : $f(\lambda)=0\}$, and the latter set is necessarily of measure zero. Thus, the Katznelson-Tzafriri assumptions impose already strong restrictions on the boundary spectrum of $T$.

The analogue of the Katznelson-Tzafriri theorem for $C_{0}$-semigroups was obtained in [ESZ92] and [Vũ92b]. We say that a function $f \in L^{1}\left(\mathbb{R}_{+}\right)$is of spectral synthesis with 
respect to a closed set $E \subset \mathbb{R}$ if there exists a sequence $\left(f_{n}\right)_{n \in \mathbb{N}} \subset L^{1}(\mathbb{R})$ such that each Fourier transform $\mathcal{F} f_{n}$ vanishes on a neighbourhood of $E$ and $\lim _{n \rightarrow \infty} f_{n}=f$ in $L^{1}(\mathbb{R})$.

Given $f \in L^{1}\left(\mathbb{R}_{+}\right)$and a bounded $C_{0}$-semigroup $(T(t))_{t \geq 0}$, we put $\hat{f}(T):=$ $\int_{0}^{\infty} f(t) T(t) d t$, the integral being strongly defined.

TheOREM 5.2. Let $A$ be the generator of a bounded $C_{0}$-semigroup $(T(t))_{t>0}$ on a $B a$ nach space $X$. If $f \in L^{1}\left(\mathbb{R}_{+}\right)$is of spectral synthesis with respect to $(-i \sigma(A)) \cap \mathbb{R}$, then $\lim _{t \rightarrow \infty}\|T(t) \hat{f}(T)\|=0$.

The following proof of Theorem 5.2, using only the simple argument from the proof of Ingham's theorem (Theorem 4.1) and the definition of spectral synthesis, shows the tauberian nature of the Katznelson-Tzafriri theorems. From the proof it is straightforward how to formulate the corresponding tauberian result for bounded functions, but we will skip this.

First proof of Theorem 5.2. Let $f$ be as in the assumption and choose $\left(f_{n}\right)_{n \in \mathbb{N}} \subset L^{1}(\mathbb{R})$ as in the definition of spectral synthesis. Convolving $f_{n}$ with appropriate test functions, we can without loss of generality assume that $\mathcal{F} f_{n}$ has compact support. Extend the semigroup by zero on the negative reals. As in the proof of Ingham's theorem, Parseval's identity implies for every $n \in \mathbb{N}$ and every $t \geq 0$

$$
\begin{aligned}
\int_{\mathbb{R}} T(t+s) f_{n}(s) d s & =\lim _{\alpha \rightarrow 0+} \int_{\mathbb{R}} e^{-\alpha(t+s)} T(t+s) f_{n}(s) d s \\
& =\lim _{\alpha \rightarrow 0+} \frac{1}{2 \pi} \int_{\mathbb{R}} R(\alpha+i \beta, A) e^{i \beta t} \mathcal{F} f_{n}(\beta) d \beta \\
& =\frac{1}{2 \pi} \int_{\mathbb{R}} R(i \beta, A) e^{i \beta t} \mathcal{F} f_{n}(\beta) d \beta .
\end{aligned}
$$

Thus, by the Lemma of Riemann-Lebesgue,

$$
\lim _{t \rightarrow \infty} \int_{\mathbb{R}} f_{n}(s) T(t+s) d s=0 .
$$

Since

$$
\lim _{n \rightarrow \infty} \int_{\mathbb{R}} f_{n}(s) T(t+s) d s=\int_{0}^{\infty} f(s) T(t+s) d s=T(t) \hat{f}(T)
$$

uniformly in $t \in \mathbb{R}_{+}$, one obtains the claim.

One may also prove the Katznelson-Tzafriri theorem by using complete trajectories. First note the following general convergence property.

Lemma 5.3. Let $(T(t))_{t \geq 0}$ be a bounded $C_{0}$-semigroup on a Banach space $X$, and let $f \in L^{1}\left(\mathbb{R}_{+}\right)$. Then $\lim _{t \rightarrow \infty} T(t) \hat{f}(T)=0$ strongly if and only if

$$
\hat{f}(T)^{*}\left(\bigcap_{t \geq 0} T^{*}(t) B^{*}\right)=\{0\} .
$$

The proof of Lemma 5.3 uses the $w^{*}$-continuity of $\hat{f}(T)^{*}$ and repeats the proof of Theorem 3.2 in [Lin71, Theorem 4.3].

Second proof of Theorem 5.2. It suffices to prove that under the assumptions of Theorem 5.2 one has $\lim _{t \rightarrow \infty} T(t) \hat{f}(T)=0$ strongly. This result applied to the semigroup defined 
by $\mathbf{T}(t) Y:=T(t) Y$ on the space of all $Y \in \mathcal{L}(X)$ such that $\lim _{t \rightarrow 0+}\|T(t) Y-Y\|=0$ yields $\lim _{t \rightarrow \infty}\|T(t) \hat{f}(T)\|=0$; compare with [Vũ92a, p. 79].

Let $f$ be as in the assumption and choose $\left(f_{n}\right)_{n \in \mathbb{N}} \subset L^{1}(\mathbb{R})$ as in the definition of spectral synthesis. As above in the first proof, we may without loss of generality assume that $\mathcal{F} f_{n}$ has compact support.

Let $F$ be a bounded complete trajectory for $\left(T(t)^{*}\right)_{t \geq 0}$. By Proposition 3.2, the Carleman spectrum of $F$ is contained in $-i \sigma(A) \cap \mathbb{R}$. By Parseval's identity,

$$
\begin{aligned}
\hat{f}(T)^{*} F(0) & =\lim _{n \rightarrow \infty} \int_{\mathbb{R}} F(t) f_{n}(t) d t=\lim _{n \rightarrow \infty} \lim _{\alpha \rightarrow 0+} \int_{\mathbb{R}} e^{-\alpha|t|} F(t) f_{n}(t) d t \\
& =\frac{1}{2 \pi} \lim _{n \rightarrow \infty} \lim _{\alpha \rightarrow 0+} \int_{\mathbb{R}}(\hat{F}(\alpha-i \beta)-\hat{F}(-\alpha-i \beta)) \mathcal{F} f_{n}(\beta) d \beta=0,
\end{aligned}
$$

where $\hat{F}$ is the Carleman transform of $F$, and the integrals are understood in the weak* sense. Since

$$
\bigcap_{t \geq 0} T^{*}(t) B^{*} \subset\left\{F(0): F \text { bounded, complete trajectory for }\left(T^{*}(t)\right)_{t \geq 0}\right\},
$$

the claim follows from Lemma 5.3.

In [ESZ90, p. 284-286] and [ESZ92, p. 215-216] it was shown how the discrete and the continuous version of the ABLV theorem (Theorem 4.4) can be deduced from the Katznelson-Tzafriri theorems. It follows from the Mittag-Leffler theorem in [Est84] that under the assumptions of the ABLV theorem $\bigcap_{\beta \in \mathbb{R}} \operatorname{Rg}(i \beta-A)$ is dense in $X$. Using this and the fact that countable closed sets are of spectral synthesis, it follows that under the assumptions of the ABLV theorem the span of $\left\{\operatorname{Rg} \hat{f}(T): f \in L^{1}\left(\mathbb{R}_{+}\right)\right.$is of spectral synthesis with respect to $-i \sigma(A) \cap \mathbb{R}\}$ is also dense in $X$ [ESZ92, Theorem 3.7].

5.2. Extensions of the Katznelson-Tzafriri theorem. Let us reconsider now the discrete Katznelson-Tzafriri theorem and discuss some extensions. First observe that the mapping $f \mapsto f(T)$ is a functional calculus from $A^{+}(\mathbb{T})$ into $\mathcal{L}(X)$ for the operator $T$. The formulation of the Katznelson-Tzafriri theorem thus suggests that for operators admitting rich functional calculi more general statements can be obtained.

For example, if $T$ is a contraction on a Hilbert space, and if $A(\mathbb{D})$ denotes the disc algebra of all holomorphic functions $\mathbb{D} \rightarrow \mathbb{C}$ which are continuous up to the boundary, then the von Neumann inequality implies that one can define a functional calculus $f \rightarrow f(T)$ from $A(\mathbb{D})$ into $\mathcal{L}(X)$ for which $\|f(T)\| \leq\|f\|_{\infty}$. Using this functional calculus, Esterle, Strouse and Zouakia proved the following ramification of the Katznelson-Tzafriri theorem in the Hilbert space setting, [ESZ90, Corollary 2.12].

THEOREM 5.4 (Esterle-Strouse-Zouakia). If $T$ is a contraction on a Hilbert space and $f \in A(\mathbb{D})$, then $\lim _{n \rightarrow \infty}\left\|T^{n} f(T)\right\|=0$ if and only if $f=0$ on $\sigma(T) \cap \mathbb{T}$.

Taking the von Neumann inequality as a starting point, it was proved in [KN97, Proposition 1.6] that Theorem 5.4 is also true for all polynomially bounded $T$.

On the other hand, it is known that contractions on Hilbert spaces even admit an $H^{\infty}(\mathbb{D})$ functional calculus. This advantageous point has been exploited by Bercovici 
[Ber90]. Recall that for every $f \in H^{\infty}(\mathbb{D})$ the radial limits $f\left(e^{i \theta}\right):=\lim _{r \rightarrow 1} f\left(r e^{i \theta}\right)$ exist almost everywhere and $\|f\|_{H^{\infty}(\mathbb{D})}=\|f\|_{L^{\infty}(\mathbb{T})}$.

THEOREM 5.5 (Bercovici). Let $T$ be a completely nonunitary contraction on a Hilbert space $H$, and let $f \in H^{\infty}(\mathbb{D})$. If $\lim _{r \rightarrow 1} f\left(r e^{i \theta}\right)=0$ for every $e^{i \theta} \in \sigma(T) \cap \mathbb{T}$ then $\lim _{n \rightarrow \infty}\left\|T^{n} f(T)\right\|=0$. The converse implication is not true.

Also Allan and Ransford obtained various generalisations of the Katznelson-Tzafriri theorem, [AR89]. In particular, for several special choices of $f$ in case when $f$ is not of spectral synthesis with respect to $\sigma(T) \cap \mathbb{T}$ they obtained sharp estimates of $\limsup _{n \rightarrow \infty}\left\|T^{n} f(T)\right\|$. This line of research was continued in [BBG96] where similar (and stronger) estimates for the strongly continuous case and for general $f$ were obtained assuming, however, countability of the boundary spectrum. Very general forms of Katznelson-Tzafriri theorems following from tauberian theorems for the vector-valued Laplace-Stieltjes transform can be found in [Bat90].

5.3. Optimality of the Katznelson-Tzafriri theorem. A natural and interesting question is whether it is possible to drop the assumption of boundedness of the operator semigroup in the Katznelson-Tzafriri theorem. Consider the special case of the discrete version of Katznelson-Tzafriri theorem with $f(z)=z-1$. This special case was in fact a motivation for the paper [KT86] and became most popular later probably because of its simple form. If $\sigma(T)=\{1\}$, the following result was obtained earlier in [Est83], and the general case can be reduced to this case as noted later in [Vũ92a]. Observe that one point sets are sets of spectral synthesis in the algebra $A(\mathbb{T})$.

Corollary 5.6 (Katznelson-Tzafriri). Let $T \in \mathcal{L}(X)$ be a power bounded operator. Then $\lim _{n \rightarrow \infty}\left\|T^{n+1}-T^{n}\right\|=0$ if and only if $\sigma(T) \cap \mathbb{T} \subset\{1\}$.

Clearly, if $\lim _{n \rightarrow \infty}\left\|T^{n+1}-T^{n}\right\|=0$ then necessarily $\lim _{n \rightarrow \infty} \frac{1}{n}\left\|T^{n}\right\|=0$ and $\sigma(T) \cap$ $\mathbb{T} \subset\{1\}$. However, are these two conditions already sufficient? If not, can they be complemented in order to obtain the same or probably a somewhat weaker conclusion?

Allan and Ransford showed that for every $\alpha>0$ there exists a Banach space operator $T$ such that $\sigma(T) \subset \mathbb{T},\left\|T^{n}\right\|=O\left(n^{\alpha}\right)$ and $\left\|T^{n}(I-T)\right\| \neq O\left(n^{\beta}\right)$ for every $\beta<\alpha$, [AR89]. Moreover, if $T$ is power bounded then $\left\|T^{n+1}-T^{n}\right\|$ may converge to zero arbitrarily slowly.

Tomilov and Zemánek provided even stronger examples, [TZ04]. It was proved that there exists a Hilbert space operator $T$ such that $\sigma(T) \cap \mathbb{T}=\{1\}, \lim _{n \rightarrow \infty} \frac{1}{n}\left\|T^{n}\right\|=0$, and $\sup _{n \geq 0}\left\|\frac{1}{n+1} \sum_{k=0}^{n} T^{k}\right\|<\infty$ (that is, $T$ is Cesàro bounded), but, at the same time, $\lim _{n \rightarrow \infty}\left\|T^{n}(T-I)^{m}\right\|=\infty$ for every $m \geq 0$. Thus, even in a Hilbert space, the additional assumption of Cesàro boundedness of $T$ does not help to get a convergence of $T^{n}$ restricted to natural subsets of $\operatorname{Rg}(T-I)$.

Answering a question of Allan, it was also proved in [TZ04] that if $X=L_{1}(0,1) \oplus$ $L_{1}(0,1)$ and

$$
T=\left(\begin{array}{cc}
I-V & -V \\
O & I-V
\end{array}\right),
$$

where $V f(t):=\int_{0}^{t} f(s) d s$ is the Volterra operator, then $\sigma(T)=\{1\}$ and $\lim _{n \rightarrow \infty} \frac{1}{n}\left\|T^{n}\right\|$ $=0$, but $\lim _{n \rightarrow \infty}\left\|T^{n}(T-I)\right\|=\infty$. Therefore, the strongest possible spectral assumption 
$\sigma(T)=\{1\}$ does not lead, in general, to the convergence of $\left\|T^{n}(T-I)\right\|$ to zero. Note, however, that in this example $\lim _{n \rightarrow \infty}\left\|T^{n}(T-I)^{5}\right\|=0$.

Hence, the Katznelson-Tzafriri theorem is optimal in several senses. The above examples show also that the corresponding tauberian theorems are optimal. In particular, this answers an open problem posed by Korevaar in [Kor02, Question 20.3].

REMARK 5.7. There is a continuous version of Corollary 5.6 due to Arendt \& Prüss [AP92, Theorem 3.10]. It says that if $(T(t))_{t \geq 0}$ is a bounded, eventually differentiable $C_{0}$-semigroup, then $\sigma(A) \cap i \mathbb{R} \subset\{0\}$ is equivalent to $\lim _{t \rightarrow \infty}\|A T(t)\|=0$. At the moment, it is not clear, whether one can find conterexamples to natural extensions of this theorem, although we strongly suspect that this is possible.

6. Resolvent conditions and stability. The boundary behaviour of the resolvent of the generator of a bounded $C_{0}$-semigroup near the imaginary axis gives important information about stability of the semigroup. However, in the previous two sections only spectral conditions were considered. By the mean ergodic theorem, they reflect some type of boundary behaviour of the resolvent near the imaginary axis, but they do this in a very rough way if one is interested in a characterisation of stability or at least more general sufficient conditions for stability. To see how far the spectral conditions and even the tauberian theorems are from a characterisation of stability one may consider the Examples 2.4 and 2.5 with appropriate weights $w$. For instance, if $w(t)=(\log (2+t))^{-1}$, then for every nonzero $f \in X_{p}$ and every $\beta \in \mathbb{R}$, the local resolvent $R(\cdot, D) f$ does not extend continuously near $i \beta$. In particular, the boundary spectrum is the whole imaginary axis, but it is even true that every nonzero orbit $S(\cdot) f$ does not satisfy the conditions neither of Ingham's tauberian theorem nor of the tauberian Theorem 4.3. Such examples show that finer resolvent conditions are needed.

Very recently, two types of such resolvent conditions have turned out to be useful: pointwise resolvent conditions reflecting the boundary behaviour of the resolvent horizontally near every point of the imaginary axis and (complex) integral resolvent conditions reflecting the boundary behaviour of integrals of local resolvents along vertical lines near the imaginary axis. Both types of conditions yield stability of semigroups on general Banach spaces, but they can become necessary and sufficient in Hilbert spaces or can be improved substantially in Banach spaces with Fourier type as we will see in Sections 8 and 9 below.

6.1. Pointwise resolvent conditions. The first statement involving pointwise resolvent conditions shows that rather mild properties of the boundary behaviour of resolvents may lead to stability of the corresponding semigroup.

THEOREM 6.1 (Pointwise resolvent condition). If A generates a bounded $C_{0}$-semigroup on a Banach space $X$ and if there exists a dense set $M \subset X$ such that for every $x \in M$ and every $\beta \in \mathbb{R}$

$$
\lim _{\alpha \rightarrow 0+} \alpha R(\alpha+i \beta, A)^{2} x=0,
$$

then the semigroup is stable. 
If $\beta \in \mathbb{R}$ and if the condition (6.1) holds for every $x \in X$, then $\left\|\alpha R(\alpha+i \beta, A)^{2}\right\| \leq M$ for all $\alpha>0$ by the uniform boundedness principle. In this case, $i \beta$ does not belong to the spectrum of $A$ since $i \beta \in \sigma(A)$ implies $\left\|\alpha^{2} R(\alpha+i \beta, A)^{2}\right\| \geq 1$ for all $\alpha>0$. Thus, the use of a dense set in Theorem 6.1 is essential as soon as the boundary spectrum of the generator is not empty.

Theorem 6.1 can be proved by using the characterisation of stability in terms of nonexistence of nontrivial, bounded, complete trajectories for the adjoint semigroup (Theorem 3.2) and an edge-of-the-wedge theorem from complex function theory from [Tom01]; see [BCT02, p. 133]. For an alternative approach via the limit isometric group see [Tom01, Theorem 4.7].

The condition (6.1) holds if for some $\gamma \in(0,1)$

$$
\lim _{\alpha \rightarrow 0+} \alpha^{\gamma} R(\alpha+i \beta, A)^{1+\gamma} x=0,
$$

or, by the mean ergodic theorem, if

$$
\lim _{\alpha \rightarrow 0+} R(\alpha+i \beta, A) x \text { exists. }
$$

Moreover, by the mean ergodic theorem again, the latter condition follows from $x \in$ $\operatorname{Rg}(i \beta-A)$ and $\overline{\operatorname{Rg}(i \beta-A)}=X$. Hence, Theorem 6.1 yields the following corollary, [BCT02, Theorem 2.4].

Corollary 6.2 (Range condition). If A generates a bounded $C_{0}$-semigroup on a Banach space $X$ and if

$$
\bigcap_{\beta \in \mathbb{R}} \operatorname{Rg}(i \beta-A) \text { is dense in } X,
$$

then the semigroup is stable.

The condition (6.2) does not, in general, characterise stability of a bounded $C_{0^{-}}$ semigroup, and even weaker conditions do not; see the comments in Section 9 below. It is an open problem whether the condition from Theorem 6.1 characterises stability of bounded $C_{0}$-semigroups, or whether on the other hand the conditions (6.1) and (6.2) are equivalent.

If $A$ satisfies the conditions of the ABLV theorem, then the condition (6.2) is satisfied by the Mittag-Leffler theorem as we saw in Section 5. Hence, the ABLV theorem is a corollary to Corollary 6.2.

We point out that spaces like the intersection of ranges in condition (6.2) are not completely new in operator theory. In fact, these spaces are very similar to so-called spectral subspaces which were already known in local spectral theory.

Given an operator (a generator) $A$, we say that a vector $x \in X$ has its local spectrum in a closed set $F \subset \mathbb{C}$ if the local resolvent $R(\cdot, A) x$ extends analytically outside $F$. When $A$ is a normal operator the set of all $x \in X$ having their local spectrum in $F \subset \mathbb{C}$, i.e. the spectral subspace corresponding to $F$, can be described algebraically as $\bigcap_{\lambda \in \mathbb{C} \backslash F} \operatorname{Rg}(\lambda-A)$, [LN00]. This description can be generalised for some classes of operators which are close to the class of normal operators. For the latest advances in this area see [MMN04]. Applications of stability theory to the study of intersections of ranges of semigroup generators are discussed in [BCT02]. 
For stability, i.e. given a generator $A$ of a bounded $C_{0}$-semigroup, the spectral subspace corresponding to the closed right half place $\bar{C}_{+}$could be of interest. Observe that every vector $x$ belonging to this spectral subspace has local spectrum in the open left half plane, i.e. the local resolvent extends analytically across the imaginary axis. By Ingham's theorem, the orbit $T(\cdot) x$ is stable for every such $x$. Hence, if the spectral subspace corresponding to the closed right half plane is dense in $X$, then the semigroup is stable.

So, it is natural to take intersections of ranges as good substitutes for spectral stability conditions. By Corollary 6.2, this strategy appears to be fruitful even when the normality of $A$ is dropped.

6.2. Integral resolvent conditions. We consider two types of integral conditions: conditions on the behaviour of the integral of local resolvents along whole vertical lines near the imaginary axis ('global integrability criterion') and conditions on the behaviour of the integral along bounded intervals of vertical lines near the imaginary axis ('local integrability criterion').

One can consider other types of integral conditions, e.g. when local resolvents belong to some Bergman space near the imaginary axis. However, these conditions can be studied by reduction to the two conditions mentioned above [CT03].

The following two results can be found in [CT03, Theorem 3.1] and in [Tom01, Theorem 4.1], respectively.

THEOREM 6.3 (Global integrability criterion). If $A$ generates a bounded $C_{0}$-semigroup on a Banach space $X$, and if for some $\gamma>1$ and for every $x$ from a dense subset of $X$

$$
\lim _{\alpha \rightarrow 0+} \int_{\mathbb{R}}\left\|\alpha^{\gamma-1} R(\alpha+i \beta, A)^{\gamma} x\right\| d \beta=0,
$$

then the semigroup is stable.

THEOREM 6.4 (Local integrability criterion). Assume that $A$ generates a bounded $C_{0}$ semigroup on a Banach space $X$. Assume also that for every $\beta \in \mathbb{R}$ there exists an open neighbourhood $U \subset \mathbb{R}$ of $\beta$ and a dense set $M \subset X$ such that

$$
\lim _{\alpha \rightarrow 0+} \int_{U}\left\|\alpha R\left(\alpha+i \beta^{\prime}, A\right)^{2} x\right\| d \beta^{\prime}=0 \quad \text { for every } x \in M
$$

Then the semigroup is stable.

It follows from Young's inequality, Plancherel's theorem and Theorem 8.1 that the condition (6.4) becomes necessary if $X$ is a Hilbert space; see the discussion in [CT03, p. 506].

It is possible to give an integral stability criterion for individual orbits of not necessarily bounded $C_{0}$-semigroups [CT03, Theorem 5.1]. It is in some sense intermediate between the similar stability condition (6.4) in Banach spaces and the integral stability criterion in Hilbert spaces in Section 8 below. 
THEOREM 6.5. Let $A$ be the generator of a $C_{0}$-semigroup $(T(t))_{t \geq 0}$ on a Banach space $X$. Let $p \in(1, \infty)$ and $q:=\frac{p}{p-1}$. Assume that

$$
\begin{aligned}
& \mathbb{C}_{+} \subset \varrho(A), \text { and } \\
& \sup _{\alpha>0} \alpha \int_{\mathbb{R}}\left\|R\left(\alpha+i \beta, A^{*}\right) x^{*}\right\|^{q} d \beta<\infty \quad \text { for every } x^{*} \in X^{*} .
\end{aligned}
$$

If, for some $x \in X$,

$$
\lim _{\alpha \rightarrow 0+} \alpha \int_{\mathbb{R}}\|R(\alpha+i \beta, A) x\|^{p} d \beta=0
$$

then

$$
\lim _{t \rightarrow \infty}\|T(t) x\|=0 .
$$

Note that under the conditions of the above theorem, the semigroup $(T(t))_{t \geq 0}$ cannot grow too fast: by [CT03, Proposition 5.3] it has a sublinear growth if $X$ has a nontrivial Fourier type.

7. Stability of positive semigroups. If more structure of the semigroup or the underlying Banach space is given, then one may expect better stability results. In this section we start to discuss such types of results, turning our attention first to positive semigroups on Banach lattices or, more generally, on ordered Banach spaces, [MN91], [Nag86], [Sch74]. Note that in general, as far as spectral or resolvent conditions for stability are concerned, the stability theory of positive semigroups comprises the same difficulties as the theory of general semigroups on Banach spaces. Examples 2.4 and 2.5 which are positive semigroups on $L^{p}$ spaces may serve as a demonstration of this; see also the discussion in Section 9 below.

Nevertheless, an interplay between positivity of a semigroup and geometric properties of the underlying Banach lattice leads to some specific stability results.

7.1. Positive semigroups on general Banach lattices. Let $X$ be a (complex) Banach lattice. The positive cone will be denoted by $X_{+}$.

We start by discussing some resolvent (resp. range) conditions for stability of positive semigroups on general Banach lattices. Recall that a Banach lattice is called a $K B$ space if every norm bounded increasing sequence converges. A Banach lattice is a KB space if and only if $c_{0} \not \subset X$.

Proposition 7.1. Let $(T(t))_{t \geq 0}$ be a bounded positive $C_{0}$-semigroup on a Banach lattice $X$.

(i) If $x \in X_{+}$and $\lim _{\alpha \rightarrow 0+} \alpha R^{2}(\alpha, A) x=0$, then $T(\cdot) x$ is stable.

(ii) If $x \in X_{+}$and $w-\lim _{\alpha \rightarrow 0+} R(\alpha, A) x$ exists, then $T(\cdot) x$ is stable.

(iii) If $x \in X_{+}, \sup _{\alpha>0}\|R(\alpha, A) x\|<\infty$, and if $X$ is a $K B$ space, then $T(\cdot) x$ is stable.

(iv) If $x \in X$ and $\lim _{\alpha \rightarrow 0+} R(\alpha, A) x_{ \pm}$exist, then $T(\cdot) x$ is stable.

Proof. (i) By positivity, $\left\|\alpha R(\alpha+i \beta, A)^{2} x\right\| \leq\left\|\alpha R(\alpha, A)^{2} x\right\|$ for every $\alpha>0, \beta \in \mathbb{R}$ and every $x \in X_{+}$. The claim thus follows from Theorem 6.1 applied to the restriction of the 
semigroup $(T(t))_{t \geq 0}$ to the closure of the linear span of $\{T(t) x: t \geq 0\}$, taking the linear span itself as a dense set; see also [Tom01, Corollary 4.9].

(ii) Since the net $(R(\alpha, A) x)_{\alpha \searrow 0}$ is increasing and weakly convergent, we obtain that $\lim _{\alpha \rightarrow 0+} R(\alpha, A) x$ exists by Dini's theorem. By the mean ergodic theorem, $\lim _{\alpha \rightarrow 0+} \alpha R(\alpha, A)^{2} x=0$, so that the assertion follows from (i). But one may also argue as follows: since $x$ is positive, the existence of $\lim _{\alpha \rightarrow 0+} R(\alpha, A) x$ implies that $\int_{0}^{\infty} T(s) x d s$ exists by the tauberian theorem [ABHN01, Theorem 4.2.16]. Now Proposition 3.10 implies the claim.

(iii) By the KB property, $\lim _{\alpha \rightarrow 0} R(\alpha, A) x$ exists. Then apply (ii).

(iv) follows directly from (ii).

By positivity, $\|R(\lambda, A) x\| \leq\|R(\operatorname{Re} \lambda, A) x\|$ for every $\lambda \in \mathbb{C}_{+}$and every $x \in X_{+}$, so that under the conditions of Proposition 7.1 (ii), (iii) or (iv) we have $\sup _{\lambda \in \mathbb{C}_{+}}\|R(\lambda, A) x\|$ $<\infty$. Thus, the conditions of Proposition 7.1 concern, in fact, the boundary behaviour of the local resolvent $R(\cdot, A) x$ in the whole right half-plane.

A result similar to Proposition 7.1 (ii) can be found in [Neu86, Proposition 3.5] and [Nag86, C-IV, Proposition 1.9].

Corollary 7.2. Let $(T(t))_{t>0}$ be a bounded positive $C_{0}$-semigroup on a Banach lattice $X$. If $\lim _{\alpha \rightarrow 0+} R(\alpha, A) x$ exists for every $x \in C^{\infty}(A):=\bigcap_{n=1}^{\infty} D\left(A^{n}\right)$, then $(T(t))_{t \geq 0}$ is stable.

For the proof it suffices to observe that $C^{\infty}(A) \cap X_{+}$is total in $X$, [Nag86, C-IV, Remark 1.10]. Corollary 7.2 has been proved in [Neu86, Proposition 3.5] with $C^{\infty}(A)$ replaced by $D(A)$, and in [Nag86, C-IV, Proposition 1.9] with $C^{\infty}(A)$ replaced by some total set $D \subset X_{+}$.

Note that under the assumptions of Corollary 7.2, much more is true. By the uniform boundedness principle (applied in the Fréchet space $C^{\infty}(A)$ ), there exists $n \in \mathbb{N}$ such that $\sup _{\alpha>0}\left\|R(\alpha, A) R\left(\lambda_{0}, A\right)^{n}\right\|<\infty$, where $\lambda_{0}>0$ is chosen large enough. Applying the resolvent identity $n$ times, this implies $\sup _{\alpha>0}\|R(\alpha, A)\|<\infty$. Hence, by positivity of $(T(t))_{t \geq 0}$, the spectral bound $s(A)<0$. By [EN99, Theorem 6.1.14], the condition $s(A)<0$ actually implies exponential stability of $T(\cdot) x$ for every $x \in D(A)$, and it even implies exponential stability of $(T(t))_{t \geq 0}$ itself if $X$ is an $L^{p}$ space, [ABHN01, Theorem 5.3.6].

By the mean ergodic theorem, the stability condition from Corollary 7.2 is equivalent to saying that $C^{\infty}(A) \subset \operatorname{Rg} A$. Clearly, it cannot be replaced by the mere condition that $\operatorname{Rg} A$ is dense in $X$ as the isometric shift semigroup on $L^{2}(\mathbb{R})$ shows. However, in some cases, the condition $\overline{\operatorname{Rg} A}=X$ characterises stability, [Nag86, C-IV Theorem 1.5].

Theorem 7.3 (Positive, norm continuous semigroups). If A generates a positive, eventually norm-continuous and bounded $C_{0}$-semigroup on a Banach lattice, then the semigroup is stable if and only if $\operatorname{Rg} A$ is dense.

Note that the eventual norm continuity of the semigroup implies $\sigma(A) \cap i \mathbb{R} \subset\{0\}$ by e.g. [Nag86, C-III Proposition 2.9, Corollary 2.13], so that Theorem 7.3 directly follows from the ABLV theorem. 
Another direction in the research on stability of positive semigroups is based on the concept of domination. There are three variants of this concept in the literature.

Definition 7.4. Let $(T(t))_{t \geq 0}$ and $(S(t))_{t \geq 0}$ be $C_{0}$ semigroups on a Banach lattice $X$ with generators $A$ and $B$, respectively. Assume that $(S(t))_{t \geq 0}$ is positive.

(i) We say that $(T(t))_{t \geq 0}$ is dominated by $(S(t))_{t \geq 0}$ if $|T(t) x| \leq S(t)|x|$ for every $x \in X$.

(ii) We say that $A$ is resolvent dominated by $B$ if $|R(\lambda, A) x| \leq R(\lambda, B)|x|$ for every $x \in X$ and every $\lambda>0$ sufficiently large.

(iii) We say that $(T(t))_{t \geq 0}$ is asymptotically dominated by $(S(t))_{t \geq 0}$ whenever $\lim _{t \rightarrow \infty}\left\|(S(t) x-T(t) x)_{-}\right\|=0$ for every positive $x \in X$, or, equivalently, $\lim _{t \rightarrow \infty} \operatorname{dist}\left(S(t) x-T(t) x, X_{+}\right)=0$.

Clearly, (i) implies (ii), and both conditions are equivalent if $X$ is $\sigma$-order complete, [Nag86, p. 269]. At the same time, (iii) is strictly weaker than (ii). Indeed, any convergent (positive) semigroup is asymptotically dominated by the constant semigroup, but clearly its generator may not be, in general, resolvent dominated by 0 , the generator of the constant semigroup.

It is natural to expect that stability properties of dominated semigroups are inherited by those of the dominating semigroups. This appears to be true even for asymptotic domination, [EKRW01, Theorem 4.5].

THEOREM 7.5. Let $X$ be a Banach lattice with order continuous norm, let $(T(t))_{t \geq 0}$ and $(S(t))_{t \geq 0}$ be positive semigroups on $X$, and assume that $(T(t))_{t \geq 0}$ is asymptotically dominated by $(S(t))_{t \geq 0}$. If $(S(t))_{t \geq 0}$ is stable, then $(T(t))_{t \geq 0}$ is also stable.

Actually, Theorem 7.5 was proved in [EKRW01] with stability replaced by convergence. If one replaces stability by convergence in Theorem 7.5, then it is shown in [EKRW01, Example 4.7 (a)] that the positivity of both semigroups, i.e. also of the dominated semigroup, is necessary in general. It is not clear whether the positivity of $(T(t))_{t \geq 0}$ is also necessary in Theorem 7.5 as we stated it here.

Inheritance of stability assuming resolvent domination seems to be more difficult to establish. However, by [RW00] (see also [RW97]), resolvent domination allows one to deduce stability of the dominated semigroup from pure spectral conditions on the generator of the dominating semigroup.

In general, one can also study stability of positive semigroups on ordered Banach spaces with a normal cone. Typical examples of ordered Banach spaces with a normal cone which are not Banach lattices are $C^{*}$-algebras. Stability of positive $C_{0}$-semigroups on $C^{*}$-algebras with unit admits a simple characterisation, [GN81, Satz 3.2].

THEOREM 7.6 (Groh-Neubrander). Let $(T(t))_{t \geq 0}$ be a positive $C_{0}$-semigroup on a $C^{*}$ algebra $\mathcal{A}$ with unit. Then $(T(t))_{t \geq 0}$ is exponentially stable, i.e. the exponential growth bound $\omega_{0}(A)<0$, if and only if it is weakly stable, i.e. $w-\lim _{t \rightarrow \infty} T(t) x=0$ for every $x \in \mathcal{A}$.

The proof of Theorem 7.6 is based on the fact that $\omega_{0}(A) \in \sigma_{p}\left(A^{*}\right)$ if $\omega_{0}(A)>-\infty$. The result is not true for $C^{*}$-algebras without unit as the example of the weakly stable left-shift semigroup on $C_{0}(\mathbb{R})$ shows. 
7.2. Positive semigroups on $L^{1}$ or $C(K)$. The $L^{1}$ space (or abstractly any $A L$ space) is distinguished from other Banach lattices by the fact that the norm is additive on the positive cone. This helps to estimate integrals of positive functions. So if a Banach lattice $X$ is an $L^{1}$-space, then Theorem 7.3 can be strengthened, [Sch74, p. 347], [Nag86, C-IV, Proposition 1.7].

Theorem 7.7 (Positive semigroups on $L^{1}$ ). If $A$ generates a positive and bounded $C_{0^{-}}$ semigroup on an $L^{1}$ space, then the semigroup is stable if and only if $\operatorname{Rg} A$ is dense.

Proof. The necessity part follows from the mean ergodic theorem. To prove the other direction, let $f \in L^{1}$ be positive. By the mean ergodic theorem,

$$
\begin{aligned}
0 & =\lim _{\lambda \rightarrow 0+}\langle\lambda R(\lambda, A) f, 1\rangle_{L^{1} \times L^{\infty}} \\
& =\lim _{\lambda \rightarrow 0+} \lambda \int_{0}^{\infty} e^{-\lambda t}\langle T(t) f, 1\rangle_{L^{1} \times L^{\infty}} d t \\
& =\lim _{\lambda \rightarrow 0+} \lambda \int_{0}^{\infty} e^{-\lambda t}\|T(t) f\|_{L^{1}} d t
\end{aligned}
$$

Passing to an equivalent norm $\|\cdot\|$ on $L^{1}$ for which $(T(t))_{t \geq 0}$ is contractive, we find that $\lim _{t \rightarrow \infty}\|T(t) f\|$ exists and $\lim _{\lambda \rightarrow 0+} \lambda \int_{0}^{\infty} e^{-\lambda t}\|T(t) f\| d t=0$. By the regularity of the Abel summation, this implies that $T(\cdot) f$ is stable for every positive $f$. Since the cone is generating, $(T(t))_{t \geq 0}$ is stable.

Concrete situations where Theorem 7.7 can be applied one may find in [ABB92] and [Bat92]. The interesting relations between domination and stability of $C_{0}$-semigroups on $L^{1}$ spaces are discussed in [Ouh97].

Stability theory of $C_{0}$-semigroups on $C(K)$, where $K$ is a compact Hausdorff space, has also several specific features. Since $C(K)$ is a (commutative) $C^{*}$-algebra with unit, Theorem 7.6 implies that a stable $C_{0}$-semigroup on $C(K)$ is necessarily exponentially stable. However, in this particular situation, more can be said.

The following result is due to Choquet \& Foiaş [CF75, Théorème 1, Remarque 5]; see [MT93, Corollaire 2.4] for a continuous version.

THEOREM 7.8. Let $(T(t))_{t \geq 0}$ be a positive $C_{0}$-semigroup on $C(K)$, and let $f \in C(K)$ be strictly positive. Then $(T(t))_{t \geq 0}$ is exponentially stable if and only if $w-\lim _{t \rightarrow \infty} T(t) f=0$.

Note that $T(t) f=M_{f}^{-1} S(t) 1$, where $M_{f} g:=f g$ and the positive $C_{0}$-semigroup $(S(t))_{t \geq 0}$ is defined by $S(t)=M_{f} T(t) M_{f}^{-1}$. Thus it suffices to prove Theorem 7.8 for $f=1$. The statement follows from the special properties of the orbit $(T(t) 1)_{t \geq 0}$ and the fact that $\|T(t)\|=\|T(t) 1\|$.

As above, Theorem 7.8 fails, if one replaces $C(K)$ by $C_{0}(\Omega)$ for some locally compact Hausdorff space $\Omega$, or if the strict positivity of $f$ is violated [CF75, Remarque 5].

The above theorem shows that it is of interest to study stability properties of $(T(t))_{t \geq 0}$ for which the orbit $T(\cdot) 1$ is not stable, e.g. for Markov semigroups (i.e. $T(t) 1=1$, $t \geq 0$ ). Note that for individual orbits of such semigroups weak stability and stability are in general not equivalent; see [Jam70, p. 369] or [CF75, Remarque 5] for illustrative examples. But these types of stability are equivalent under the additional assumption of irreducibility of $(T(t))_{t \geq 0}$. 
Given a $C_{0}$-semigroup $(T(t))_{t \geq 0}$ of contractions on $C(K)$, we call a set $E \subset K$ invariant if for every $x \in E$ and every $t \geq 0$ we have $\operatorname{supp} T(t)^{*} \delta_{x} \subset E$. The semigroup is called irreducible on $C(K)$ if the only invariant subsets are $K$ and the empty set. This definition of irreducibility is equivalent to the one for positive semigroups on general Banach lattices (see [Sch74, Nag86] for that definition).

The following statement was proved by Jamison in the context of Markov operators, [Jam70]. Its generalisation for nonpositive operators was obtained by Sine in [Sin89, Theorem 2]. We state the continuous version which can be derived from the discrete one easily.

THEOREM 7.9 (Sine). Let $(T(t))_{t \geq 0}$ be an irreducible contraction $C_{0}$-semigroup on $C(K)$, and let $f \in C(K)$. Then $T(\cdot) f$ is stable if and only if $w-\lim _{t \rightarrow \infty} T(t) f=0$.

The example from [Jam70, p. 369] shows that the condition of irreducibility cannot be omitted in Theorem 7.9 even if $(T(t))_{t \geq 0}$ is a Markov semigroup. Moreover, the statement fails if $C(K)$ is replaced by $C_{0}(\Omega)$. However, weaker notions of irreducibility still allow one to keep the conclusion of Theorem 7.9; we refer to [Wit88] for details. Concerning stability and convergence of irreducible semigroups on $L_{1}$ spaces see also [Nag86, p. 346-349].

There is another kind of condition which is suitable for the study of convergence of Markov operators [Rub77, Theorem 12].

THEOREM 7.10 (Rubinov). Let $T$ be a Markov operator on $C(K)$. Assume that the fixed points of $T$ separate the points of $K$. Then $\left(T^{n}\right)_{n \geq 0}$ is convergent if and only if it is mean ergodic.

By means of the abstract Theorem 3.2, various other stability conditions for Markov operators were obtained in [Lin74]. We will not state them here, since their formulation would require probabilistic terms going far beyond the scope of the survey. Recently, the convergence properties of Markov operators were also investigated from a measuretheoretic point of view in [Fog99].

8. Stability of semigroups on Hilbert spaces. Stability of semigroups on Hilbert spaces is perhaps the nicest part of the theory of stability. The special geometric properties of Hilbert spaces, e.g. the validity of Plancherel's theorem, and of operators on Hilbert spaces (normal operators, hyponormal operators, contractions on Hilbert spaces) have lead to a variety of stability results, some of which are even characterising stability in terms of resolvent conditions.

Moreover, the study of stability of semigroups on Hilbert spaces is of independent interest in operator theory, e.g. for the invariant subspace problem. However, despite several partial positive results it is still not known whether a stable $T \in \mathcal{L}(X)$ with nonempty boundary spectrum has a nontrivial invariant subspace; see [Mül03], [Mül05]. The invariant subspace problem is also open for the opposite class of power bounded $T$ for which all orbits are not stable, [Kér89], [KV03] (if, however, $T$ and $T^{*}$ are non-stable, then it is known that $T$ (and $T^{*}$ ) has an invariant subspace, [SNF70, Theorem II.5.4]).

This may serve as an indication that stable operator semigroups are still not fully understood. Note that stability theory and invariant subspace theory have many methods 
and techniques in common. As illustrative works we mention [Atz84], [Bea88], [AE98] and [Kér99].

We start with a discussion of resolvent stability conditions. The following statement is a refinement of the global integrability criterion from Theorem 6.3. Its particular case with $\gamma=1$ has been proved in [Tom01, Theorem 3.1].

THEOREM 8.1 (Global integrability criterion). Let $A$ be the generator of a bounded $C_{0^{-}}$ semigroup on a Hilbert space $X$. Then the semigroup is stable if and only if for some $\gamma>\frac{1}{2}$ and every $x$ from a dense subset of $X$,

$$
\lim _{\alpha \rightarrow 0+} \int_{\mathbb{R}}\left\|\alpha^{\gamma-\frac{1}{2}} R(\alpha+i \beta, A)^{\gamma} x\right\|^{2} d \beta=0 .
$$

Proof. By changing the norm on $X$, we may without loss of generality assume that the semigroup $(T(t))_{t>0}$ generated by $A$ is a contraction semigroup. Note that $X$ need not be a Hilbert space for the new norm, but it is isomorphic to a Hilbert space, and the Fourier transform is still an isomorphism on $L^{2}(\mathbb{R} ; X)$ by Plancherel's theorem.

If $(T(t))_{t \geq 0}$ is a contraction semigroup, then $\lim _{t \rightarrow \infty}\|T(t) x\|$ exists for every $x \in X$. By an abelian theorem and Plancherel's theorem,

$$
\begin{aligned}
\lim _{t \rightarrow \infty}\|T(t) x\|^{2} & =\lim _{\alpha \rightarrow 0} \int_{0}^{\infty} \alpha^{2 \gamma-1} t^{2 \gamma-2} e^{-2 \alpha t}\|T(t) x\|^{2} d t \\
& \leq C \lim _{\alpha \rightarrow 0} \int_{\mathbb{R}}\left\|\alpha^{\gamma-\frac{1}{2}} R(\alpha+i \beta, A)^{\gamma} x\right\|^{2} d \beta .
\end{aligned}
$$

This proves the necessity part. The sufficiency part is proved similarly.

One can use the characterisation of generators of bounded semigroups on Hilbert spaces due to Gomilko [Gom99, Theorem 1] and Shi \& Feng [SF00, Theorem 1.1, Theorem 4.1] in order to drop the boundedness condition on the semigroup in Theorem 8.1 and to replace it by a pure resolvent condition.

THEOREM 8.2. Let $A$ be a closed, densely defined, linear operator on $X$ such that $\mathbb{C}_{+} \subset$ $\varrho(A)$, and let $\gamma \in\left(\frac{1}{2}, 2\right)$. Then $A$ generates a stable semigroup if and only if

$$
\sup _{\alpha>0} \int_{\mathbb{R}}\left\|\alpha^{\frac{3}{2}-\gamma} R\left(\alpha+i \beta, A^{*}\right)^{2-\gamma} x\right\|^{2} d \beta<\infty,
$$

and for every $x \in X$

$$
\lim _{\alpha \rightarrow 0+} \int_{\mathbb{R}}\left\|\alpha^{\gamma-\frac{1}{2}} R(\alpha+i \beta, A)^{\gamma} x\right\|^{2} d \beta=0 .
$$

The conditions (8.2) and (8.3) are clearly necessary by the Plancherel theorem and an argument similar to that of the proof of Theorem 8.1. For the proof of their sufficiency, observe that (8.2) and (8.3) imply

$$
\sup _{\alpha>0} \alpha \int_{\mathbb{R}}\left|\left\langle R^{2}(\alpha+i \beta, A) x, y\right\rangle\right| d \beta<\infty, \quad x, y \in X,
$$

by Young's inequality. Then, by [Gom99] or [SF00], $A$ generates a bounded semigroup, and we obtain the stability of $(T(t))_{t \geq 0}$ from Theorem 8.1.

Using Theorem 8.2 with $\gamma=1$, Guo \& Zwart have shown that it provides a geometric stability criterion for $C_{0}$-semigroups with generator $A$ in terms of solvability of the 
Lyapunov equations

$$
\begin{aligned}
& (A-s I)^{*} Q(s)+Q(s)(A-s I)=-I \text { on } D(A) \text { and } \\
& (A-s I) \tilde{Q}(s)+\tilde{Q}(s)(A-s I)^{*}=-I \text { on } D\left(A^{*}\right)
\end{aligned}
$$

for all $s>0$; see [GZ06, Theorem 2.8]. Their result is somewhat parallel to the well known characterisation of exponential stability of semigroups in terms of Lyapunov equations.

TheOREM 8.3 (Guo-Zwart). Let $A$ be a closed, densely defined, linear operator on a Hilbert space $X$. Then the following statements are equivalent.

(i) A generates a stable $C_{0}$-semigroup.

(ii) For every $s>0$ there exist positive solutions $Q(s), \tilde{Q}(s) \in \mathcal{L}(X)$ of the Lyapunov equations (8.4) and (8.5), respectively, such that

(a) $\sup \{\|s Q(s)\|,\|s \tilde{Q}(s)\|: s>0\}<\infty$ and

(b) $\lim _{s \rightarrow 0+}\langle s Q(s) x, x\rangle=0$ for every $x \in H$.

The pointwise resolvent condition as well as the corresponding range stability condition from Sections 6 and 9 can be improved for semigroups on Hilbert space as the following statement shows.

THEOREM 8.4 (Pointwise criterion). Let $A$ be the generator of a bounded $C_{0}$-semigroup on a Hilbert space $X$.

(i) If there exists a dense set $M \subset X$ such that

$$
\lim _{\alpha \rightarrow 0+} \sqrt{\alpha} R(\alpha+i \beta, A) x=0 \text { for every } x \in M \text { and every } \beta \in \mathbb{R},
$$

then the semigroup is stable.

(ii) If

$$
\bigcap_{\beta \in \mathbb{R}} \operatorname{Rg}(i \beta-A)^{\frac{1}{2}} \text { is dense in } X,
$$

then the semigroup is stable.

Theorem 8.4 (i) was obtained in [Tom01, Theorem 3.4] by using the method of limit isometric groups; we give a different proof in Section 9 below (Theorem 9.1). Theorem 8.4 (ii) is a consequence of part (i) and can be found in [CT03, Proposition 6.1]. Note that the range condition (8.7) is weaker than the range condition (6.2) from Theorem 6.2 ; this can be seen by considering Examples 2.4 and 2.5, [CT03]. Also the condition (8.6) is a priori not stronger than the corresponding Banach space condition (6.1) by the abstract Hardy-Landau inequality, [Tom01, Remark 4.11]. It is an open problem whether the stability conditions from Theorem 8.4 (i) or (ii) are also necessary for stability.

Contractions on Hilbert spaces have a rich spectral theory based on unitary dilations and functional model approaches. So the results given above can be improved and complemented for the class of contraction semigroups.

Recall that if $(T(t))_{t \geq 0}$ is a $C_{0}$-semigroup of contractions on a Hilbert space $H$, then there is a unitary $C_{0}$-group $(U(t))_{t \in \mathbb{R}}$ on a Hilbert space $K \supset H$ such that $P_{H} U(t)=$ $T(t), t \geq 0$, where $P_{H}$ is the orthogonal projection onto $H$. Any such group $(U(t))_{t \in \mathbb{R}}$ is 
called a dilation of $(T(t))_{t \geq 0}$, and among all dilations there exists a minimal one in the sense that $K=\bigvee_{t \in \mathbb{R}} U(t) \bar{H}$.

Foguel expressed stability and convergence of a $C_{0}$-semigroup $(T(t))_{t \geq 0}$ of contractions in geometric terms by means of the corresponding minimal unitary dilation $(U(t))_{t \in \mathbb{R}}$, [Fog71, Corollary 1, Theorem 4]. His characterisation of stability and convergence is very similar to the characterisation in terms of limit isometric groups or complete trajectories. For a discussion of relations between limit isometric semigroups and minimal unitary dilations see [Kér89]. Let $H_{\infty}:=\bigcap_{t \geq 0} \bigvee_{t>s} U(-s) H$.

THEOREM 8.5. A contraction semigroup on a Hilbert space $H$ is stable if and only if $H_{\infty}=\{0\}$. Moreover, the semigroup is convergent if and only if the minimal unitary dilation is constant on $H_{\infty}$.

In [Put75, Theorem 3] a stability result for cohyponormal operators is proved, i.e. for operators $T$ on a Hilbert space such that $T T^{*}-T^{*} T \geq 0$. Note that a cohyponormal power bounded operator is necessarily a contraction. A short proof of the following statement has been given in [Oku77] and [KV94].

THEOREM 8.6 (Putnam). If $T$ is a completely nonunitary cohyponormal contraction, then $\left(T^{n}\right)_{n \geq 0}$ is stable.

Putnam's approach to Theorem 8.6 is of interest here since it follows ideas similar to those behind the pointwise resolvent conditions from this survey. We present a variant of his approach using a short argument due to Radjabalipour [Rad76]. Observe that for every $z \in \mathbb{C}$

$$
(T-z)\left(T^{*}-\bar{z}\right) \geq(T-z)\left(T^{*}-\bar{z}\right)-\left(T^{*}-\bar{z}\right)(T-z) \geq T T^{*}-T^{*} T:=D^{2} \geq 0,
$$

where the last inequality holds by the cohyponormality of $T$. By the Douglas majorization criterion [Dou66], for every $z \in \mathbb{C}$ there exists a contraction $C(z)$ such that

$$
(T-z) C(z)=D \text {. }
$$

The global boundedness of $C(\cdot)$ is used in [Put75] to show that $\operatorname{Rg} D \subset\{x \in X$ : $\left.\lim _{n \rightarrow \infty} T^{n} x=0\right\}$. Since $T$ is completely nonunitary, $\operatorname{Rg} D$ is dense in $X$ and therefore $\left(T^{n}\right)_{n \geq 0}$ is stable.

Note that the equation (8.8) alone together with the density of $\operatorname{Rg} D$ already suffices to obtain stability of $\left(T^{n}\right)_{n \geq 0}$ by, for example, Corollary 6.2.

If $A$ is the generator of a semigroup of completely nonunitary contractions, then the condition (8.6) characterises stability, but even a little bit more is true. This characterisation of stability has been proved in [Tom01, p. 75-76], but see also [BL95, Theorem 5] where the characterisation is proved with a concrete set $M$. The proof in [Tom01] is based on Theorem 8.5 together with some additional properties of the orthogonal projection onto $H_{\infty}$ found in [Fog71]. It is crucial that the generator of the minimal unitary dilation of a semigroup of completely nonunitary contractions has absolutely continuous spectral measure.

THEOREM 8.7. Let $A$ be the generator of a $C_{0}$-semigroup of completely nonunitary contractions on a Hilbert space $X$. Then the following are true: 
(i) The semigroup is stable if and only if there exists a dense set $M \subset X$ such that

$$
\lim _{\alpha \rightarrow 0+} \sqrt{\alpha} R(\alpha+i \beta, A) x=0 \text { for every } x \in M \text { and almost every } \beta \in \mathbb{R} \text {. }
$$

(ii) If there exists a set $E \subset \mathbb{R}$ of measure 0 such that

$$
\bigcap_{\beta \in \mathbb{R} \backslash E} \operatorname{Rg}(i \beta-A)^{\frac{1}{2}} \text { is dense in } X,
$$

then the semigroup is stable.

(iii) If the boundary spectrum of $A$ has measure 0 , then the semigroup is stable.

As in Theorem 8.4, part (ii) is a consequence of part (i) and can be proved along the lines of [CT03, Corollary 6.1]. The last statement of Theorem 8.7 can already be found in [SNF70, Proposition 6.7, p. 85]. It can be used to deduce a discrete version of the ABLV theorem for Hilbert space contractions, [Gil70, Proposition 2]. At the end, we turn to a variational condition for stability.

Theorem 8.8. Let $A$ be densely defined, closed linear operator on a Hilbert space $X$. If $A$ is normal, and if

$$
\operatorname{Re}(A x, x)<0 \text { for all } x \in D(A),
$$

then $A$ generates a stable $C_{0}$-semigroup of contractions.

If $A$ is a multiplication operator on an $L^{2}$ space, then the above theorem is easy to prove. Thus, the theorem is an immediate consequence of the spectral theorem for (unbounded) normal operators on separable Hilbert spaces ([RS80, Theorem VIII.4, p. 260]) saying that $A$ is unitarily equivalent to a multiplication operator.

The normality assumption on $A$ in Theorem 8.8 can be dropped if one knows that the semigroup generated by $A$ is asymptotically almost periodic or if $A$ has compact resolvent. See [ABHN01, p. 360-361] for the relevant discussion of these two properties of $A$.

In Djačenko [Dja80], Theorem 8.8 is stated without the assumption that $A$ is normal, but then it becomes false as the following example shows.

EXAmPle 8.9. Consider the shift semigroup $(S(t))_{t \geq 0}$ from Example 2.4 on the Hilbert space $X=L^{2}\left(\mathbb{R}_{+} ; w(t) d t\right)$, but with weight $w(t)=1+e^{-t}, t \geq 0$. A simple integration by parts shows that the generator $D=-d / d t$ of this semigroup satisfies the condition (8.9). However, for every nonzero $f \in X$ one has $\limsup _{t \rightarrow \infty}\|S(t) f\|>0$.

Other examples of this kind can be found in [BL95]. However, if a closed, densely defined linear operator $A$ satisfies (8.9), then there is another Hilbert space $K$ containing $X$ such that $A$ generates a stable semigroup on $K$, [BL95, Theorem 2].

9. Stability of semigroups on Banach spaces with Fourier type. In many of the stability results up to now, especially those based on spectral conditions or resolvent conditions, the Fourier transform has played a central role in the proofs. For example, in the proofs of the tauberian theorems which we have presented (Ingham's theorem and its variants) Parseval's identity and the Lemma of Riemann-Lebesgue were the core. In some of the stability results for semigroups on Hilbert spaces, the validity of Plancherel's 
theorem for Hilbert space valued $L^{2}$ functions was essential, and since, by Plancherel, the Fourier transform is an isomorphism on $L^{2}$, we even obtained some characterisations of stability in terms of resolvent conditions. Heuristically, the reason why all these arguments work is that the Fourier transform of bounded semigroup orbits is the distributional boundary value of the corresponding local resolvents on the imaginary axis or the unit circle, respectively.

It is thus not surprising that in Banach spaces in which the Fourier transform preserves good geometric properties we are able to obtain better stability results, at least better than the pointwise and the integral stability results from Section 6. Among such Banach spaces are the Banach spaces with nontrivial Fourier type.

We say that a Banach space $X$ has Fourier type $p \in[1,2]$ if the Fourier transform on $\mathcal{S}(\mathbb{R} ; X)$ (the vector-valued Schwartz space) extends to a bounded linear operator from $L^{p}(\mathbb{R} ; X)$ into $L^{q}(\mathbb{R} ; X)$, i.e. if the Hausdorff-Young inequality holds. By the Lemma of Riemann-Lebesgue, every Banach space $X$ has the trivial Fourier type $p=1$. Thus, by interpolation, if $X$ has Fourier type $p \in[1,2]$, then it has Fourier type $p^{\prime}$ for every $p^{\prime} \in[1, p]$. A Banach space has Fourier type $p=2$ (i.e. Plancherel's Theorem holds) if and only if it is isomorphic to a Hilbert space. By [Bou82], a Banach space has nontrivial Fourier type if and only if it is B-convex; see also [Bou82] for a definition of B-convexity.

If a Banach space $X$ has Fourier type $p \in[1,2]$, then the dual $X^{*}$ has the same Fourier type $p$. For all these facts we refer to the recent survey [GKKT98].

9.1. Pointwise resolvent conditions. The first result is in the spirit of the pointwise resolvent conditions from Theorem 6.1 (Banach space case) and Theorem 8.4 (i) (Hilbert space case), and it has been proved in [BCT05, Theorem 5.7]. It is an improvement of [CT04, Theorem 4.2]. Note that Theorem 8.4 (i) is Theorem 9.1 in the case $p=2$.

THEOREM 9.1 (Pointwise resolvent condition). Let $A$ be the generator of a bounded $C_{0^{-}}$ semigroup on a Banach space $X$ having Fourier type $p \in(1,2]$. Let $q:=\frac{p}{p-1}$ be the conjugate exponent. Assume that there exists a dense set $M \subset X$ such that for every $\beta \in \mathbb{R}$ and every $x \in M$

$$
\lim _{\alpha \rightarrow 0+}\left\|\alpha^{\frac{1}{q}} R(\alpha+i \beta, A) x\right\|=0 .
$$

Then the semigroup is stable.

The proof of Theorem 9.1 presented below is very similar to the proof of [BCT02, Theorem 6.3].

Proof of Theorem 9.1. Denote the semigroup by $\mathcal{T}$. Let $F: \mathbb{R} \rightarrow X^{*}$ be a bounded complete trajectory for the adjoint semigroup $\mathcal{T}^{*}$, let $x \in M$, and let $f:=\langle F, x\rangle$. Let $\hat{F}$ and $\hat{f}$ be the Carleman transforms of $F$ and $f$, respectively.

By the resolvent identity (3.1) (Proposition 3.5), for every $\alpha>0$ and every $\beta \in \mathbb{R}$,

$$
\begin{aligned}
|\hat{f}(\alpha+i \beta)-\hat{f}(-\alpha+i \beta)| & =\left|2\left\langle\alpha^{\frac{1}{p}} \hat{F}(-\alpha+i \beta), \alpha^{\frac{1}{q}} R(\alpha+i \beta, A) x\right\rangle\right| \\
& \leq G(\alpha+i \beta) H(\alpha+i \beta),
\end{aligned}
$$

where

$$
G(\alpha+i \beta):=\left\|2 \alpha^{\frac{1}{p}} \hat{F}(-\alpha+i \beta)\right\|
$$


and

$$
H(\alpha+i \beta):=\left\|\alpha^{\frac{1}{q}} R(\alpha+i \beta, A) x\right\| .
$$

By the boundedness of $F$ and the Hausdorff-Young inequality,

$$
\sup _{\alpha>0}\|G(\alpha+i \cdot)\|_{L^{q}(\mathbb{R})}<\infty .
$$

Moreover, from the assumption (9.1), the resolvent identity and the boundedness of the semigroup $(T(t))_{t \geq 0}$ we obtain for every $\theta_{0} \in\left(0, \frac{\pi}{2}\right)$ and every $\beta \in I$

$$
\begin{aligned}
& \limsup _{\substack{\alpha \rightarrow 0+\\
\left|\beta^{\prime}-\beta\right| \leq \alpha \tan \theta_{0}}} H\left(\alpha+i \beta^{\prime}\right) \\
\leq & \limsup _{\substack{\alpha \rightarrow 0+\\
\left|\beta^{\prime}-\beta\right| \leq \alpha \tan \theta_{0}}}\left\|\alpha^{\frac{1}{q}}\left(R\left(\alpha+i \beta^{\prime}, A\right)-R(\alpha+i \beta, A)\right) x\right\| \\
\leq & \limsup _{\substack{\alpha \rightarrow 0+\\
\left|\beta^{\prime}-\beta\right| \leq \alpha \tan \theta_{0}}}\left\|\alpha \tan \theta_{0} R\left(\alpha+i \beta^{\prime}, A\right) \alpha^{\frac{1}{q}} R(\alpha+i \beta, A) x\right\| \\
\leq & \tan \theta_{0} \sup _{t \geq 0}\|T(t)\| \limsup _{\alpha \rightarrow 0+}\left\|\alpha^{\frac{1}{q}} R(\alpha+i \beta, A) x\right\|=0 .
\end{aligned}
$$

It follows from this inequality, the boundedness of $F$ and (9.2) that we can apply Theorem 2.3 in order to see that the Carleman transform $\hat{f}$ extends analytically through the imaginary axis to an entire function.

By [Prü93, Proposition 0.5 (i)] and the uniqueness of the Fourier transform, $f=$ $\langle F, x\rangle=0$. Since $M$ is dense in $X$, this implies $F=0$, i.e. there is no nontrivial bounded complete trajectory for $\mathcal{T}^{*}$. By Theorem 3.2, the semigroup $\mathcal{T}$ is stable.

As a corollary to Theorem 9.1 one obtains the following, [BCT05, Corollary 5.10], [CT04, Corollary 4.6].

Corollary 9.2 (Range condition). Let $A$ be the generator of a bounded $C_{0}$-semigroup on a Banach space $X$ having Fourier type $p \in(1,2]$. If

$$
\bigcap_{\beta \in \mathbb{R}} \operatorname{Rg}(i \beta-A)^{\frac{1}{p}} \text { is dense in } X \text {, }
$$

then the semigroup is stable.

9.2. Optimality of pointwise resolvent conditions. It has been shown in [CT03, Section 4] that the Corollaries 6.2 and 9.2 are optimal in the following senses.

If one considers the isometric (nonstable) shift group with generator $D$ on $X=L^{q}(\mathbb{R})$ $(1<q<\infty)$ or on $X=C_{0}(\mathbb{R})$, then, by [CT03, Proposition 4.10], for every $1<q \leq \infty$ and every $\gamma \in\left(0, \frac{q-1}{q}\right)$ the space

$$
\bigcap_{\beta \in \mathbb{R}} \operatorname{Rg}(i \beta-A)^{\gamma} \text { is dense in } X \text {. }
$$

If $q \geq 2$, then the space $X$ has Fourier type $p:=\frac{q}{q-1}$. This example shows that one cannot expect better exponents (better than the exponent $\gamma=\frac{1}{p}$ ) in Corollary 9.2.

On the other hand, while the conditions (6.2) and (9.3) cannot not be improved, in the sense that the exponent $\gamma=\frac{1}{p}$ cannot be chosen smaller, they are in general also not necessary for stability. If $D$ is the generator of the stable right-shift semigroup 
from Example 2.4 on the space $X_{p}=L^{p}\left(\mathbb{R}_{+} ; w(t) d t\right)(1 \leq p<\infty)$ with weight $w(t)=$ $(\ln (e+t))^{-1}$, then for every $\gamma \in\left(\frac{p-1}{p}, 1\right]$ and every interval $J \subset \mathbb{R}$

$$
\bigcap_{\beta \in J} \operatorname{Rg}(i \beta-D)^{\gamma}=\{0\} .
$$

Thus, if $p \in[1,2)$, then $X_{p}$ has Fourier type $p$ and $\frac{1}{p}>\frac{p-1}{p}$. By (9.4), the condition (9.3) in Corollary 9.2 is not satisfied, [CT03, Proposition 4.3].

Similarly, in Example 2.5, for the same choice of the weight $w$ and the parameter $p$, one has

$$
\bigcap_{\beta \in J} \operatorname{Rg}(i \beta-D) \text { is not dense in } X_{p}
$$

see [CT03, Proposition 4.7]. In this example, $D$ generates even a $C_{0}$-group of sublinear growth and is a generalized scalar.

Note that these examples only show that range conditions of the types (6.2) and (9.3) are not necessary for stability if the Fourier type is smaller than 2. As already mentioned above, these examples do not seem to provide counterexamples for the possibility that the range condition (8.7) is necessary for stability of semigroups in Hilbert spaces.

9.3. Integral resolvent conditions. The integral resolvent conditions from Section 6 can be improved in Banach spaces with nontrivial Fourier type [CT03, Theorem 3.1, Theorem 3.3]. The proof of the first stability result follows the lines of the proof of the corresponding statement in Theorem 8.1, replacing Plancherel's theorem by the Hausdorff-Young inequality or the Lemma of Riemann-Lebesgue. The proof of the second stability result is very similar to the proof of Theorem 6.4 and is based on Theorem 3.2.

THEOREM 9.3 (Global integrability criterion). Let $A$ be the generator of a bounded $C_{0^{-}}$ semigroup on a Banach space $X$ having Fourier type $p \in[1,2]$. If, for some $\gamma>\frac{1}{p}$ and for every $x$ from a dense subset of $X$,

$$
\lim _{\alpha \rightarrow 0+} \int_{\mathbb{R}}\left\|\alpha^{\gamma-\frac{1}{p}} R(\alpha+i \beta, A)^{\gamma} x\right\|^{p} d \beta=0,
$$

then the semigroup is stable.

THEOREM 9.4 (Local integrability criterion). Let $A$ be the generator of a bounded $C_{0^{-}}$ semigroup on a Banach space $X$ having Fourier type $p \in(1,2]$. Let $q$ be the conjugate exponent. Assume that for every $\beta \in \mathbb{R}$ there exists an open neighbourhood $U \subset \mathbb{R}$ of $\beta$ and a dense set $M \subset X$ such that

$$
\lim _{\alpha \rightarrow 0+} \int_{U}\left\|\alpha^{\frac{1}{q}} R\left(\alpha+i \beta^{\prime}, A\right) x\right\|^{p} d \beta^{\prime}=0 \quad \text { for every } x \in M .
$$

Then the semigroup is stable.

We remark that there are other geometric properties of Banach spaces which can also be of value for stability theory. The relevance of the analytic Radon-Nikodym property was shown in [Chi98] and [HN99], and the more general analytic Riemann-Lebesgue property was introduced and applied to the study of stability in [BC02]. We refer to these papers for the corresponding definitions and precise statements. 
We remark at the end of this section that B-convex Banach spaces in connection with stability of individual orbits of semigroups have also been studied in [HN99] and [Wro99].

10. Stability of evolution semigroups. Particularly interesting for applications are the so-called evolution semigroups. They help to study qualitative properties of evolution families which are usually associated with nonautonomous abstract Cauchy problems of the form

$$
u^{\prime}(t)=A(t) u(t), t \geq s \geq 0, \quad u(s)=x .
$$

We call a family $(U(t, s))_{t \geq s \geq 0} \subset \mathcal{L}(X)$ an evolution family if $U(t, t)=I, U(t, s)=$ $U(t, r) U(r, s)$ and if $U(\cdot, \cdot) x$ is continuous for all $t \geq r \geq s \geq 0$ and all $x \in X$.

Wellposedness of the problem (10.1) should actually be equivalent to the existence of an evolution family the orbits of which are the unique mild solutions of (10.1). However, we emphasise that the notion of mild solutions of the Cauchy problem (10.1) is not uniquely defined in the literature and it is in some cases not satisfactory. We will not go into details here and will just assume that an evolution family is given.

If such an evolution family $(U(t, s))_{t \geq s \geq 0}$ on a Banach space $X$ is exponentially bounded, then

$$
\left(\mathcal{T}_{p}(t) f\right)(s)=\left\{\begin{array}{ll}
U(s, s-t) f(s-t), & s \geq t, \\
0, & s<t,
\end{array} \quad t, s \geq 0, f \in E_{p},\right.
$$

defines a $C_{0}$-semigroup on $E_{p}:=L^{p}\left(\mathbb{R}_{+} ; X\right)(1 \leq p<\infty)$, and on $E_{\infty}:=C_{00}\left(\mathbb{R}_{+} ; X\right)$ (the space of continuous functions vanishing at 0 and at infinity). That semigroup is called the evolution semigroup associated with $(U(t, s))_{t \geq s \geq 0}$. We denote by $\mathcal{G}_{p}$ its generator.

It is known that the evolution semigroups reflect qualitative properties of the corresponding evolution family. For example, exponential stability or exponential dichotomy of an evolution family can be characterised in terms of exponential stability or exponential dichotomy of the associated evolution semigroup. Since the spectral mapping theorem holds for evolution semigroups, one can even characterise exponential stability or exponential dichotomy of evolution families in terms of the location of the spectrum of the generator of the associated evolution semigroup. For all these results, we refer to [CL99].

The fact that also mere stability of evolution families can be characterised by stability of the associated evolution semigroups has been proved in [BCT02, Theorem 2.2]. Note that we say that an evolution family $(U(t, s))_{t \geq s \geq 0}$ is stable if $\lim _{t \rightarrow \infty} U(t, s) x=0$ for all $s \in \mathbb{R}_{+}$and all $x \in X$.

In Theorem 10.1 below we call a function $F: \mathbb{R}_{-} \rightarrow X^{*}$ a complete trajectory for the evolution family $\left(U(-s,-t)^{*}\right)_{s \leq t \leq 0}$ if $U(-s,-t)^{*} F(s)=F(t)$ for all $s \leq t \leq 0$. This definition of a complete trajectory differs from the corresponding definition in Section 3.2 in that $F$ is only defined on the half-line $\mathbb{R}_{-}$. However, in the autonomous case, i.e. when $U(t, s)=T(t-s)$ for some $C_{0}$-semigroup $(T(t))_{t \geq 0}$, a complete trajectory for $\left(U(-s,-t)^{*}\right)_{s \leq t \leq 0}$ can be uniquely extended to a complete trajectory for $\left(T(t)^{*}\right)_{t \geq 0}$ on $\mathbb{R}$ by defining $F(t)=T(t)^{*} F(0)$ for $t \geq 0$.

THEOREM 10.1. Let $(U(t, s))_{t \geq s \geq 0}$ be a bounded evolution family on a Banach space $X$, and let $\left(\mathbf{T}_{p}(t)\right)_{t \geq 0}$ be the evolution semigroup associated with $(U(t, s))_{t \geq s \geq 0}$ on $E_{p}$ 
$(1 \leq p \leq \infty)$. Then the following assertions are equivalent:

(1) The evolution family $(U(t, s))_{t \geq s \geq 0}$ is stable.

(2) If $B^{*}$ denotes the unit ball in $X^{*}$, then the set

$$
J^{*}:=\bigcup_{s \geq 0} \bigcap_{t \geq s} U(t, s)^{*}\left(B^{*}\right)
$$

is trivial, i.e. $J^{*}=\{0\}$.

(3) The evolution family $\left(U(-s,-t)^{*}\right)_{s \leq t \leq 0}$ does not admit a bounded nontrivial complete trajectory.

(4) The semigroup $\left(\mathbf{T}_{p}(t)\right)_{t \geq 0}$ is stable for some $1 \leq p \leq \infty$.

(5) The semigroup $\left(\mathbf{T}_{p}(t)\right)_{t \geq 0}$ is stable for all $1 \leq p \leq \infty$.

(6) $\operatorname{Rg} \mathcal{G}_{1}$ is dense in $L^{1}\left(\mathbb{R}_{+} ; X\right)$.

(7) The set

$$
F:=\left\{f \in L^{1}\left(\mathbb{R}_{+} ; X\right): U * f \in L^{1}\left(\mathbb{R}_{+} ; X\right)\right\}
$$

is dense in $L^{1}\left(\mathbb{R}_{+} ; X\right)$, where $(U * f)(t):=\int_{0}^{t} U(t, \tau) f(\tau) d \tau, t \in \mathbb{R}_{+}$.

The equivalences $(1) \Leftrightarrow(2) \Leftrightarrow(3)$ generalise Theorem 3.2 to the case of bounded evolution families. The equivalence $(1) \Leftrightarrow(6)$ is based on the observation that $\operatorname{Ker} \mathcal{G}_{1}^{*}$ consists of bounded complete trajectories for $\left(U^{*}(-s,-t)\right)_{t \geq s}$. Thus stability of $(U(t, s))_{t \geq s \geq 0}$ is equivalent to the density of $\operatorname{Rg} \mathcal{G}_{1}$. It is not too difficult to show that $\operatorname{Rg} \mathcal{G}_{1}=F$ and then the equivalence $(6) \Leftrightarrow(7)$ is clear.

Note that the equivalence $(1) \Leftrightarrow(7)$ looks similar to Datko's characterisation of exponential stability saying that $(U(t, s))_{t \geq s \geq 0}$ is exponentially stable if and only if the set $F$ from $(7)$ is equal to $L^{1}\left(\mathbb{R}_{+} ; X\right)$. However, while in Datko's theorem one may replace $p=1$ by any $1 \leq p \leq \infty$, one cannot do this in Theorem 10.1 (7). For example, if $X$ is reflexive and $p \in(1, \infty)$, then the set

$$
\left\{f \in L^{p}\left(\mathbb{R}_{+} ; X\right): U * f \in L^{p}\left(\mathbb{R}_{+} ; X\right)\right\}
$$

is always dense in $L^{p}\left(\mathbb{R}_{+} ; X\right)$, due to the mean ergodic theorem.

The other equivalent statements are interesting for the study of stability of evolution families in terms of stability of semigroups. We also point out that the equivalence $(5) \Leftrightarrow(6)$ looks very similar to the stability condition for positive semigroups on $L^{1}$ spaces (Theorem 7.7).

\section{References}

[AB88] W. Arendt and C. J. K. Batty, Tauberian theorems and stability of one-parameter semigroups, Trans. Amer. Math. Soc. 306 (1988), 837-852.

[AB97] S. Ansari and P. Bourdon, Some properties of cyclic operators, Acta Sci. Math. (Szeged) 63 (1997), 195-207.

[ABB92] W. Arendt, C. J. K. Batty, and Ph. Bénilan, Asymptotic stability of Schrödinger semigroups, Math. Z. 209 (1992), 511-518.

[ABHN01] W. Arendt, C. J. K. Batty, M. Hieber, and F. Neubrander, Vector-valued Laplace Transforms and Cauchy Problems, Monographs in Mathematics 96, Birkhäuser, Basel, 2001. 
[AE98] S. Ansari and P. Enflo, Extremal vectors and invariant subspaces, Trans. Amer. Math. Soc. 350 (1998), 539-558.

[AM04] C. Ambrozie and V. Müller, Invariant subspaces for polynomially bounded operators, J. Funct. Anal. 213 (2004), 321-345.

[AP92] W. Arendt and J. Prüss, Vector-valued tauberian theorems and asymptotic behavior of linear Volterra equations, SIAM J. Math. Anal. 23 (1992), 412-448.

[AR89] G. R. Allan and T. J. Ransford, Power-dominated elements in a Banach algebra, Studia Math. 94 (1989), 63-79.

[ARS04a] A. Aleman, S. Richter, and C. Sundberg, Analytic contractions and nontangential boundary behavior, in: Proceedings of the first advanced course in operator theory and complex analysis, Sevilla, 2004.

[ARS04b] A. Aleman, S. Richter, and C. Sundberg, Invariant subspaces for the backward shift on Hilbert spaces of analytic functions with regular norms, preprint, 2004.

[Atz84] A. Atzmon, On the existence of hyperinvariant subspaces, J. Operator Theory 11 (1984), 1-40.

[Bal60] A. V. Balakrishnan, Fractional powers of closed operators and the semigroups generated by them, Pacific J. Math. 10 (1960), 419-437.

[Bas79] A. G. Baskakov, Spectral criteria for almost periodicity of solutions of functional equations, Math. Notes (Engl. transl.) 24 (1979), 606-612.

[Bas95] B. Basit, Some problems concerning different types of vector valued almost periodic functions, Dissertationes Math. 338 (1995).

[Bas97] B. Basit, Harmonic analysis and asymptotic behavior of solutions to the abstract Cauchy problem, Semigroup Forum 54 (1997), 58-74.

[Bat90] C. J. K. Batty, Tauberian theorems for the Laplace-Stieltjes transform, Trans. Amer. Math. Soc. 322 (1990), 783-804.

[Bat92] C. J. K. Batty, Asymptotic stability of Schrödinger semigroups: path integral methods, Math. Ann. 292 (1992), 457-492.

[Bat94] C. J. K. Batty, Asymptotic behaviour of semigroups of operators, in: Banach Center Publications 30, Polish Acad. Sci., Warsaw, 1994, 35-52.

[Bat96] C. J. K. Batty, Spectral conditions for stability of one-parameter semigroups, J. Differential Equations 127 (1996), 87-96.

[BBG96] C. J. K. Batty, Z. Brzeźniak, and D. A. Greenfield, A quantitative asymptotic theorem for contraction semigroups with countable unitary spectrum, Studia Math. 121 (1996), 167-182.

[BC02] $\mathrm{S} . \mathrm{Bu}$ and $\mathrm{R}$. Chill, Banach spaces with the Riemann-Lebesgue or the analytic Riemann-Lebesgue property, Bull. London Math. Soc. 34 (2002), 569-581.

[BCT02] C. J. K. Batty, R. Chill, and Y. Tomilov, Strong stability of bounded evolution families and semigroups, J. Funct. Anal. 193 (2002), 116-139.

[BCT05] A. Borichev, R. Chill and Y. Tomilov, Uniqueness theorems for (sub-)harmonic functions with applications to operator theory, preprint, 2005.

[Bea88] B. Beauzamy, Introduction to Operator Theory and Invariant Subspaces, NorthHolland Mathematical Library 42, North-Holland, Amsterdam, 1988.

[Ber90] H. Bercovici, On the iterates of a completely nonunitary contraction, in: Topics in Operator Theory: Ernst D. Hellinger memorial volume, Operator Theory Adv. Appl. 48, Birkhäuser, Basel, 1990, 185-188.

[Ber93] H. Bercovici, Commuting power-bounded operators, Acta Sci. Math. (Szeged) 57 (1993), 55-64. 
[BG94] C. J. K. Batty and D. A. Greenfield, On the invertibility of isometric semigroup representations, Studia Math. 110 (1994), 235-250.

[BL95] K. N. Boyadzhiev and N. Levan, Strong stability of Hilbert space contraction semigroups, Stud. Sci. Math. Hung. 30 (1995), 165-182.

[BNR98a] C. J. K. Batty, J. M. A. M. van Neerven, and F. Räbiger, Local spectra and individual stability of uniformly bounded $C_{0}$-semigroups, Trans. Amer. Math. Soc. 350 (1998), 2071-2085.

[BNR98b] C. J. K. Batty, J. M. A. M. van Neerven, and F. Räbiger, Tauberian theorems and stability of solutions of the Cauchy problem, Trans. Amer. Math. Soc. 350 (1998), 2087-2103.

[Bou82] J. Bourgain, A Hausdorff-Young inequality for B-convex Banach spaces, Pacific J. Math. 101 (1982), 255-262.

[Car44] T. Carleman, L'intégrale de Fourier et questions qui s'y rattachent, Publications Scientifiques de l'Institut Mittag-Leffler 1, Uppsala, 1944.

[CF75] J. G. Choquet and C. Foiaş, Solution d'un problème sur les itérés d'un opérateur positif sur $C(K)$ et propriétés de moyennes associées, Ann. Inst. Fourier 25 (1975), 109-129.

[CF02] R. Chill and E. Fašangová, Equality of two spectra arising in harmonic analysis and semigroup theory, Proc. Amer. Math. Soc. 130 (2002), 675-681.

[Chi98] R. Chill, Tauberian theorems for vector-valued Fourier and Laplace transforms, Studia Math. 128 (1998), 55-69.

[CL99] C. Chicone and Y. Latushkin, Evolution Semigroups in Dynamical Systems and Differential Equations, Mathematical Surveys and Monographs 70, Amer. Math. Soc., Providence, R.I., 1999.

[CP01] R. Chill and J. Prüss, Asymptotic behaviour of linear evolutionary integral equations, Integral Eq. Operator Theory 39 (2001), 193-213.

[CT03] R. Chill and Y. Tomilov, Stability of $C_{0}$-semigroups and geometry of Banach spaces, Math. Proc. Cambridge Phil. Soc. 135 (2003), 493-511.

[CT04] R. Chill and Y. Tomilov, Analytic continuation and stability of operator semigroups, J. Analyse Math. 93 (2004), 331-358.

[Dat72] R. Datko, Uniform asymptotic stability of evolutionary processes in a Banach space, SIAM J. Math. Anal. 3 (1972), 428-445.

[Der76] Y. Derriennic, Lois "zéro ou deux" pour les processus de Markov. Applications aux marches aléatoires, Ann. Inst. Henri Poincaré Sect. B 12 (1976), 111-129.

[Dix50] J. Dixmier, Les moyennes invariantes dans les semi-groupes et leurs applications, Acta Sci. Math. (Szeged) 12 (1950), 213-227.

[Dja80] S. V. Djačenko, Asymptotic stability of semigroups with a strictly dissipative generating operator, Mat. Zametki 28 (1980), 75-78 (in Russian).

[DL01] Y. Derriennic and M. Lin, Fractional Poisson equations and ergodic theorems for fractional coboundaries, Israel J. Math. 123 (2001), 93-130.

[Dou66] R. G. Douglas, On majorization, factorization, and range inclusion of operators on Hilbert space, Proc. Amer. Math. Soc. 17 (1966), 413-415.

[Dou69] R. G. Douglas, On extending commutative semigroups of isometries, Bull. London Math. Soc. 1 (1969), 157-159.

[Dra70] D. Drasin, An application of Tauberian methods to a problem in series, Amer. Math. Monthly 77 (1970), 152-156. 
[dLVW02] R. deLaubenfels, Quôc Phong Vũ, and S. Wang, Stability of semigroups of operators and spectral subspaces, Semigroup Forum 64 (2002), 337-354.

[EKRW01] E. Yu. Emel'yanov, U. Kohler, F. Räbiger, and M. Wolff, Stability and almost periodicity of asymptotically dominated semigroups of positive operators, Proc. Amer. Math. Soc. 129 (2001), 2633-2642.

[EN99] K. J. Engel and R. Nagel, One-Parameter Semigroups for Linear Evolution Equations, Graduate Texts in Mathematics 194, Springer, Berlin, 1999.

[Est83] J. Esterle, Elements for a classification of commutative radical Banach algebras, in: Radical Banach Algebras (Long Beach, 1981), Lect. Notes Math. 975, Springer, 1983, 4-65.

[Est84] J. Esterle, Mittag-Leffler methods in the theory of Banach algebras and a new approach to Michael's problem, in: Proc. Conference on Banach Algebras and Several complex variables (New Haven, Conn., 1983), Contemp. Math. 32, Amer. Math. Soc., Providence, RI, 1984, 107-129.

[ESZ90] J. Esterle, E. Strouse, and F. Zouakia, Theorems of Katznelson-Tzafriri type for contractions, J. Funct. Anal. 94 (1990), 273-287.

[ESZ92] J. Esterle, E. Strouse, and F. Zouakia, Stabilité asymptotique de certains semigroupes d'opérateurs et idéaux primaires de $L^{1}\left(\mathbb{R}_{+}\right)$, J. Operator Theory 28 (1992), 203-227.

[Fog71] S. R. Foguel, Some geometric aspects of unitary dilation of a contraction operator, Rev. Roumaine Math. Pures Appl. 16 (1971), 671-675.

[Fog99] S. R. Foguel, Weak and strong convergence of the iterates of a Markov operator, Houston J. Math. 25 (1999), 121-131.

[GKKT98] J. García-Cuerva, K. S. Kazaryan, V. I. Kolyada, and Kh. L. Torrea, The HausdorffYoung inequality with vector-valued coefficients and applications, Uspekhi Mat. Nauk 53 (1998), 3-84 (in Russian); English transl.: Russian Math. Surveys 53 (1998), 435-513.

[Gha75] P. Ghatage, Iterates of an absolutely continuous operator, Proc. Amer. Math. Soc. 49 (1975), 203-204.

[Gil70] F. Gilfeather, The structure and asymptotic behavior of polynomially compact operators, Proc. Amer. Math. Soc. 25 (1970), 127-134.

[GM04] E. G. Guttierrez and A. Montes, The Volterra operator is not supercyclic, Integral Eq. Operator Theory 50 (2004), 211-216.

[GN81] U. Groh and F. Neubrander, Stabilität starkstetiger, positiver Operatorhalbgruppen auf $C^{*}$-Algebren, Math. Ann. 256 (1981), 509-516.

[Gom99] A. M. Gomilko, On conditions for the generating operator of a uniformly bounded $C_{0}$-semigroup of operators, Funktsional. Anal. i Prilozhen. 33 (1999), 66-69 (in Russian); English transl.: Funct. Anal. Appl. 33 (1999), 294-296.

[GZ06] B. Z. Guo and H. Zwart, On the relation between stability of continuous and discrete-time evolution equations via the Cayley transform, Integral Eq. Operator Theory 54 (2006), 349-383.

[Haa06] M. Haase, The Functional Calculus for Sectorial Operators, Birkhäuser Verlag, Basel, 2006.

[HN99] S.-Z. Huang and J. M. A. M. van Neerven, B-convexity, the analytic RadonNikodym property and individual stability of $C_{0}$-semigroups, J. Math. Anal. Appl. 231 (1999), 1-20.

[HP57] E. Hille and R. S. Phillips, Functional Analysis and Semi-Groups, Amer. Math. Soc., Providence, R.I., 1957. 
[Jam70] B Jamison, Irreducible Markov operators on $C(S)$, Proc. Amer. Math. Soc. 24 (1970), 366-370.

[Kér89] L. Kérchy, Isometric asymptotes of power bounded operators, Indiana Univ. Math. J. 38 (1989), 173-188.

[Kér94] L. Kérchy, Unitary asymptotes of Hilbert space operators, in: Banach Center Publications 30, Polish Acad. Sci., Warsaw, 1994, 191-201.

[Kér99] L. Kérchy, Hyperinvariant subspaces of operators with non-vanishing orbits, Proc. Amer. Math. Soc. 127 (1999), 1363-1370.

[Kér05] L. Kérchy, Cyclic properties and stability of commuting power bounded operators, Acta Sci. Math. (Szeged) 71 (2005), 299-312.

[Kat68] Y. Katznelson, An Introduction to Harmonic Analysis, Wiley, New York, 1968.

[KN97] L. Kérchy and J. M. A. M. van Neerven, Polynomially bounded operators whose spectrum on the unit circle has measure zero, Acta Sci. Math. (Szeged) 63 (1997), 551-562.

[Kom66] H. Komatsu, Fractional powers of operators, Pacific J. Math. 19 (1966), 285-346.

[Kom69] H. Komatsu, Fractional powers of operators IV: potential operators, J. Math. Soc. Japan 21 (1969), 221-228.

[Kor02] J. Korevaar, A century of complex tauberian theory, Bull. Amer. Math. Soc. (N.S.) 39 (2002), 475-531.

[KT86] Y. Katznelson and L. Tzafriri, On power bounded operators, J. Funct. Anal. 68 (1986), 313-328.

[Kub97] C. S. Kubrusly, An Introduction to Models and Decompositions in Operator Theory, Birkhäuser, Basel, 1997.

[KV94] C. S. Kubrusly and P. C. M. Vieira, Strong stability for cohyponormal operators, J. Operator Theory 31 (1994), 123-127.

[KV03] L. Kérchy and Quôc Phong Vũ, On invariant subspaces for power-bounded operators of class $C_{1}$, Taiwanese J. Math. 7 (2003), 69-75.

[Lin71] M. Lin, Mixing of Markov operators, Z. Wahrscheinlichkeitstheorie verw. Geb. 19 (1971), 231-242.

[Lin74] M. Lin, Convergence of the iterates of a Markov operator, Z. Wahrscheinlichkeitstheorie verw. Geb. 29 (1974), 153-163.

[LN00] K. B. Laursen and M. M. Neumann, An Introduction to Local Spectral Theory, London Mathematical Society Monographs 20, Oxford University Press, Oxford, 2000.

[LSPL03] F. León-Saavedra and A. Piqueras-Lerena, Cyclic properties of Volterra operator, Pacific J. Math. 211 (2003), 157-162.

[LV88] Yu. I. Lyubich and Quôc Phong Vũ, Asymptotic stability of linear differential equations in Banach spaces, Studia Math. 88 (1988), 37-42.

[LZ82] B. M. Levitan and V. V. Zhikov, Almost Periodic Functions and Differential Equations, Cambridge University Press, 1982.

[Mül88] V. Müller, Local spectral radius formula for operators in Banach spaces, Czechoslovak Math. J. 38 (1988), 726-729.

[Mül01] V. Müller, Orbits, weak orbits and local capacity of operators, Integral Equations Operator Theory 41 (2001), 230-253.

[Mül03] V. Müller, Power bounded operators and supercyclic vectors, Proc. Amer. Math. Soc. 131 (2003), 3807-3812. 
[Mül05] V. Müller, Power bounded operators and supercyclic vectors II, Proc. Amer. Math. Soc. 133 (2005), 2997-3004.

[McI86] A. McIntosh, Operators which have an $H^{\infty}$ functional calculus, Proc. Centre Math. Anal. Austr. Nat. Univ. 14 (1986), 210-231.

[MMN04] T. L. Miller, V. G. Miller, and M. M. Neumann, Spectral subspaces of subscalar and related operators, Proc. Amer. Math. Soc. 132 (2004), 1483-1493.

[MN91] P. Meyer-Nieberg, Banach Lattices, Springer, Berlin, 1991.

[MS01] C. Martinez and M. Sanz, The Theory of Fractional Powers of Operators, NorthHolland Mathematical Studies 187, Elsevier, Amsterdam, 2001.

[MT93] E. Ménard and J.-P. Troallic, Semigroupes de contractions linéaires sur $C(X)$ : irréductibilité et théorèmes de convergence, Semigroup Forum 47 (1993), 87-95.

[Nag86] R. Nagel (ed.), One-parameter Semigroups of Positive Operators, Lect. Notes in Math. 1184, Springer, 1986.

[Nee96] J. M. A. M. van Neerven, The Asymptotic Behaviour of Semigroups of Linear Operators, Operator Theory: Advances and Applications 88, Birkhäuser, 1996.

[Nee02] J. M. A. M. van Neerven, Lower semicontinuity and the theorem of Datko and Pazy, Integral Eq. Operator Theory 42 (2002), 482-492.

[Neu86] F. Neubrander, Laplace transform and asymptotic behaviour of strongly continuous semigroups, Houston J. Math. 12 (1986), 549-561.

[Oku77] K. Okubo, The unitary part of paranormal operators, Hokkaido Math. J. 6 (1977), 273-275.

[Ouh97] E. M. Ouhabaz, Growth and asymptotics of perturbed recurrent semigroups, Semigroup Forum 55 (1997), 160-166.

[Prü93] J. Prüss, Evolutionary Integral Equations and Applications, Monographs in Mathematics 87, Birkhäuser, Basel, 1993.

[Put75] C. R. Putnam, Hyponormal contractions and strong power convergence, Pacific J. Math. 57 (1975), 531-538.

[Rad76] M. Radjabalipour, On majorization and normality of operators, Proc. Amer. Math. Soc. 62 (1976), 105-110.

[RS80] M. Reed and B. Simon, Methods of Modern Mathematical Physics, I: Functional Analysis. Second edition, Academic Press, New York, 1980.

[Rub77] A. M. Rubinov, Sublinear operators and their applications, Uspehi Mat. Nauk 32 (1977), no. 4(196), 113-174, 287.

[RW97] F. Räbiger and M. P. H. Wolf, Spectral and asymptotic properties of dominated operators, J. Austral. Math. Soc. Ser. A 63 (1997), 16-31.

[RW00] F. Räbiger and M. P. H. Wolf, Spectral and asymptotic properties of resolventdominated operators, J. Austral. Math. Soc. Ser. A 68 (2000), 181-201.

[Sch57] L. Schwartz, Théorie des distributions à valeurs vectorielles I, Ann. Inst. Fourier 7 (1957), 1-141.

[Sch58] L. Schwartz, Théorie des distributions à valeurs vectorielles II, Ann. Inst. Fourier 8 (1958), 1-208.

[Sch74] H. H. Schaefer, Banach Lattices and Positive Operators, Grundlehren der mathematischen Wissenschaften 215, Springer, Berlin, 1974.

[SF00] D.-H. Shi and D.-X. Feng, Characteristic conditions of the generation of $C_{0}$ semigroups in a Hilbert space, J. Math. Anal. Appl. 247 (2000), 356-376.

[Sin89] R. Sine, Weakly constricted operators and Jamison's convergence theorem, Proc. Amer. Math. Soc. 106 (1989), 751-755. 
[SN47] B. Sz.-Nagy, On uniformly bounded linear transformations in Hilbert space, Acta Sci. Math. (Szeged) 11 (1947), 152-157.

[SNF70] B. Sz.-Nagy and C. Foiaş, Harmonic Analysis of Operators on Hilbert Space, NorthHolland, Amsterdam, and Akadémiai Kiadó, Budapest, 1970.

[SS82] G. M. Sklyar and V. Y. Shirman, On the asymptotic stability of a linear differential equation in a Banach space, Teor. Funktsiǔ Funkts. Anal. Prilozh. 37 (1982), 127132, (in Russian).

[Tom01] Y. Tomilov, A resolvent approach to stability of operator semigroups, J. Operator Theory 46 (2001), 63-98.

[TZ04] Y. Tomilov and J. Zemánek, A new way for constructing examples in operator ergodic theory, Math. Proc. Cambridge Phil. Soc. 137 (2004), 209-225.

[Vũ92a] Quôc Phong Vũ, A short proof of the Y. Katznelson's and L. Tzafriri's theorem, Proc. Amer. Math. Soc. 115 (1992), 1023-1024.

[Vũ92b] Quôc Phong Vũ, Theorems of Katznelson-Tzafriri type for semigroups of operators, J. Funct. Anal. 119 (1992), 74-84.

[Vũ93] Quôc Phong Vũ, On the spectrum, complete trajectories and asymptotic stability of linear semidynamical systems, J. Differential Equations 105 (1993), 30-45.

[Vũ97] Quôc Phong Vũ, Almost periodic and strongly stable semigroups of operators, in: Linear Operators, J. Zemánek (ed.), Banach Center Publ. 38, Polish Acad. Sci., 1997, 401-427.

[Wes98] U. Westphal, A generalized version of the abelian mean ergodic theorem with rates for semigroup operators and fractional powers of infinitesimal generators, Result. Math. 34 (1998), 381-394.

[Wit88] R. Wittmann, Schwach irreduzible Markoff-Operatoren, Monatsh. Math. 105 (1988), 319-334.

[Wol47] F. Wolf, Extension of analytic functions, Duke Math. J. 14 (1947), 877-887.

[Wro99] V. Wrobel, Optimal individual stability estimates for $C_{0}$-semigroups in Banach spaces, Trans. Amer. Math. Soc. 351 (1999), 4981-4994.

[Yos78] K. Yosida, Functional Analysis, Springer, Berlin, 1978. 
